\title{
Synthesis and Structure-Activity Relationship Study of
}

\section{Potent Cytotoxic Analogs of the Marine Alkaloid}

\section{Lamellarin D}

Daniel Pla, ${ }^{a}$ Antonio Marchal, ${ }^{a \|}$ Christian A. Olsen, ${ }^{a, \mathbb{1}}$ Andrés Francesch, ${ }^{b}$ Carmen Cuevas, ${ }^{b}$

$$
\text { Fernando Albericio, }{ }^{a, *^{*}} \text { Mercedes Álvarez }{ }^{a, \S, *}
$$

a: Institute for Research in Biomedicine, Barcelona Science Park-University of Barcelona, Josep Samitier 1-5 , 08028 Barcelona, Spain

E-08028 Barcelona, Spain; b: Pharma Mar, Avda Reyes Católicos 1, E- 28770 Colmenar Viejo, Madrid, Spain

dpla@pcb.ub.es, albericio@pcb.ub.es, $\underline{\text { malvarez@pcb.ub.es }}$

|| Current address: Department of Inorganic and Organic Chemistry, University of Jaén, 23071 Jaén; amarchal@ujaen.es

${ }^{\Uparrow}$ Current address: The Danish University of Pharmaceutical Sciences, Copenhagen, Denmark cao@dfuni.dk

${ }^{\ddagger}$ Department of Organic Chemistry, University of Barcelona, 08028 Barcelona

$\S$ Laboratory of Organic Chemistry, Faculty of Pharmacy, University of Barcelona, 08028 Barcelona

* Fernando Albericio, Tel: (+34) 93403 7088; Mercedes Álvarez, Tel: (+34) 93403 7086; Fax: (+34)

934037126 
Title running head: Synthesis and Structure-Activity Relationship of Analogs of Lam-D

\title{
RECEIVED DATE (to be automatically inserted after your manuscript is accepted if required
}

\begin{abstract}
The marine alkaloid, Lamellarin D (Lam-D), has shown potent cytotoxicity in numerous cancer cell lines, and was recently identified as a potent topoisomerase I inhibitor. A library of open lactone analogs of Lam-D was prepared from a methyl 5,6-dihydropyrrolo[2,1-a]isoquinoline-3carboxylate scaffold (1) by introducing various aryl groups through sequential and regioselective bromination, followed by $\mathrm{Pd}(0)$-catalyzed Suzuki cross-coupling chemistry. The compounds were obtained in a $24-44 \%$ overall yield, and tested in a panel of three human tumor cell lines, MDA-MB231 (breast), A-549 (lung), and HT-29 (colon), to evaluate their cytotoxic potential. From these data the SAR study concluded that more than $75 \%$ of the open-chain Lam-D analogs tested showed cytotoxicity in a low micromolar $\mathrm{GI}_{50}$ range.
\end{abstract}

Marine alkaloids, cytotoxic activity, heterocycles, cross-coupling reactions

\section{INTRODUCTION}

In the search for new bioactive, small chemical molecules for research in chemical biology and medicinal chemistry one must choose a starting point from the vast chemical space. ${ }^{1}$ In this respect, natural products may serve as biologically pre-validated leads, ${ }^{2,3}$ and indeed more than $60 \%$ of the recently marketed drugs have been isolated from natural products or synthetic compounds based on natural products. ${ }^{4}$ With the recent advances in natural products science, including the synthesis of complex libraries, ${ }^{2,3}$ biosynthesis, ${ }^{5}$ and isolation techniques, ${ }^{6,7}$ the field has a promising future. ${ }^{8}$ In particular, marine and microbial environments may serve as a source of new bioactive chemical compounds. ${ }^{9}$

Here we used Lamellarin D (Lam-D, Figure 1), a potent cytotoxic agent against various tumor cells, as a lead. This marine alkaloid was first isolated from the marine prosobranch mollusc Lamellaria sp. 
in 1985 by Faulkner and coworkers. ${ }^{10}$ Since then a family of about 35 structurally related lamellarins has been isolated from natural sources, and several synthetic strategies have been devised for these natural products. ${ }^{11,12}$ Of the family of lamellarins, Lam-D is one of the most potent lead candidates for anticancer chemotherapy. There is substantial evidence that Lam-D is an inhibitor of topoisomerase $\mathrm{I}^{13}$ and a potent pro-apoptotic agent. ${ }^{14}$ Recently, topoisomerase I binding studies have been elaborated further by comparing Lam-D and Camptothecin ${ }^{15}$ (Figure 1) bound to the DNA-topoisomerase I complex using molecular dynamics simulations. ${ }^{16}$ These also correlate nicely with structure-activity relationships (SAR) obtained with homologs of Lam-D with distinct $\mathrm{OMe} / \mathrm{OH}$ substitution patterns on the pentacyclic framework. ${ }^{16,17}$ Hence, the $8-\mathrm{OH}$ and 20-OH groups (see Figure 1) are crucial for cytotoxic activity and also for topoisomerase I inhibition.

Moreover, the unsaturated C-5-C-6 motif of Lam-D, as compared to the saturated analog (Lam-501, Figure 1), is important for potency, ${ }^{13,18}$ a trend that was also observed with a range of derivatives of Lam-D and Lam-501, in which the free phenolic sites were acylated. ${ }^{18}$ Furthermore, the latter study afforded potent candidates for in vivo preclinical development of their anti-tumor activity. Interestingly, derivatization of the $8-\mathrm{OH}$ and $20-\mathrm{OH}$ groups with amino acids, thus preserving the hydrogen bonding capacity at these sites, affords potent compounds, while acylation with various carboxylic acids results in a considerable decrease in potency. ${ }^{18}$

We recently reported preliminary biological results showing that simplified tricyclic analogs of LamD lacking the lactone, such as Open Lam-501 (Figure 1), retain some cytotoxic activity. ${ }^{19}$ This finding encouraged us to perform SAR studies using scaffold $\mathbf{1}$ by incorporating various aryl groups in positions 1 and 2, including their oxidized homologs (Figure 1). ${ }^{20}$

In addition to the initial achievements in the assembly of the pentacyclic lamellarin framework ${ }^{21-23}$ and total synthesis of Lam-D, ${ }^{21}$ pentacyclic and more simple lamellarins have been synthesized using solid-phase synthesis, ${ }^{24-26}$ which should facilitate the preparation of compound libraries for biological evaluation. However, here we found it more rational to prepare our library using the methyl 5,6dihydropyrrolo[2,1-a]isoquinoline-3-carboxylate scaffold 1 (Figure 1), and protocols developed for 
modular total synthesis of Lam- $\mathrm{D}^{27}$ and tricyclic analogs. ${ }^{19}$ While this study was in progress, another highly efficient synthesis of Lam-D and related analogs was published. ${ }^{28}$

\section{RESULTS AND DISCUSSION}

\section{Chemistry}

The synthesis of an open-chain lamellarin analogs library was performed in solution starting from the methyl pyrrole-2-carboxylate by transformation into scaffold $1 .{ }^{19,27}$ The key steps in the process were the introduction of the aryl substituents on positions 1 and 2 of the scaffold using the boron derivatives 4 and $\mathbf{5}$ as building blocks for the final structure. Following the procedure described for the total synthesis of Lam-D, ${ }^{27}$ the synthetic strategy used consisted of the regioselective bromination of the scaffold followed by a $\operatorname{Pd}(0)$-catalyzed Suzuki cross-coupling reaction, oxidation and subsequent deprotection of all the phenols present in each compound. The isopropyl ether was used as protecting group for the phenols present in the final compounds and was maintained throughout the synthetic process. $^{29}$

Three alternative ways were used to introduce the aryl groups on scaffold 1, according the final structure of the lamellarin analogs (Scheme 1). The monoaryl compounds 6 were prepared by regioselective bromination of scaffold $\mathbf{1}$ on position 1 to give the bromoderivative $\mathbf{2}$, which was used for Suzuki cross-coupling with the boronic acids 4. Diarylderivatives 7 with the same substitution pattern in both aryl groups were obtained from the dibromo-scaffold $\mathbf{3}$ by simultaneous introduction of both aryl groups. Finally, for the diarylated-compounds 9, with different substituents on the phenyl rings, we used two sequential regioselective bromination and cross-coupling reactions starting from scaffold $\mathbf{1}$ with the monoaryl-scaffolds $\mathbf{6}$ and bromides $\mathbf{8}$ as synthetic intermediates. ${ }^{27}$

An extensive range of aryl boronic derivatives 4 and 5 were used as building blocks (see Table 1 for the structures). Building blocks 4 are commercially available ${ }^{30}$ whereas the ortho-substituted borolanes 5 were obtained in good yields (52-81\%) from the proper aryl bromide by $\mathrm{Pd}(0)$-catalyzed cross-coupling borylation using the pinacolborane, as described in the supporting information.$^{27,31}$ 
All the Suzuki cross-coupling reactions between bromides 2, 3 and 8 and building blocks 4 were performed in DMF using $\mathrm{Pd}\left(\mathrm{PPh}_{3}\right)_{4}$ and $\mathrm{K}_{2} \mathrm{CO}_{3}$ as catalyst and base, respectively, with good yields. The phenolic group on position 4' of $\mathbf{6 c}\left(\mathrm{R}^{4}=\mathrm{OH}\right)$ was protected as isopropoxy-ether by reaction with 2bromopropane in basic conditions, thereby giving $\mathbf{6 d} .^{32}$ Generally, transformation of $\mathbf{6}$ into $\mathbf{8}$ was performed using $N$-bromosuccinimide (NBS) in tetrahydofuran (THF) with a careful control of the reaction time in order to obtain the desired mono- and regio-bromination, thereby avoiding the formation of complex mixtures. ${ }^{33}$ Regioselective bromination of electron-rich systems like $\mathbf{6 h}, \mathbf{6}$, and 6n using the same reaction conditions was unsuccessful because halogenation on the electron-rich aromatic ring could not be avoided with these compounds. ${ }^{34}$ The Suzuki reaction conditions used to introduce the second aryl ring on $\mathbf{8}$ were basically the same as when the boron-derivatives $\mathbf{4}$ were used. However, with the more hindered borolanes 5, several modification were required such as the slow addition of three equivalent ${ }^{35}$ of $\mathbf{5}$ and the use of $\mathrm{K}_{3} \mathrm{PO}_{4}$ as base to afford yields between $81 \%$ and quantitative for the second cross-coupling (see experimental conditions). ${ }^{36}$ Compounds 9a-i were prepared by reaction of scaffolds $\mathbf{8}$ and the second building block $\mathbf{5}$, as indicated in Table 1 and in the experimental conditions.

Optimization of oxidation was performed with the 2-thienylderivative $4 \mathbf{n}$. Several experiments using 2,3-dichloro-5,6-dicyano-p-benzoquinone (DDQ) in $\mathrm{CHCl}_{3}$ at reflux temperature, $\mathrm{MnO}_{2}$ in refluxing toluene or pyridine, ${ }^{37}$ or Pd-C in toluene or decalin ${ }^{38}$ afforded only traces of $\mathbf{1 0 n}$. The best reaction conditions were attained using DDQ in $\mathrm{CHCl}_{3}$ as solvent in a sealed tube and with microwave (MW) irradiation. The aromatization of dihydroisoquinolines 6,7 and 9 to give the planar system of pyrrolo[2,1-a]isoquinoline present in compounds 10-12 was accomplished using the same protocol as described in the supporting information. ${ }^{39}$ The ${ }^{1} \mathrm{H}-\mathrm{NMR}$ was crucial for the control of the reaction because the dihydroisoquinolines 6-9 have a characteristic ABXY spin system for the four protons of $\mathrm{C}^{5} \mathrm{H}_{2}$ and $\mathrm{C}^{6} \mathrm{H}_{2}$ while the isoquinolines 10-12 hold an $\mathrm{AB}$ system in the aromatic area for the two protons $\mathrm{C}^{5} \mathrm{H}$ and $\mathrm{C}^{6} \mathrm{H}$, the former being a significant signal. 
Compounds 9f-i and 12f-i both with bulky substituents in ortho of the aryl rings, showed restricted rotation and two conformers were observed by ${ }^{1} \mathrm{H}$ - and ${ }^{13} \mathrm{C}-\mathrm{NMR} .{ }^{1} \mathrm{H}-\mathrm{NMR}$ experiments with $\mathbf{1 2 f}$ at variable temperature showed the collapse of the signals at $75{ }^{\circ} \mathrm{C}$ (see Figure 2 in the Supporting Information). As an example, the coalescence of double doublets at 6.29 and $6.32 \mathrm{ppm}^{40}$ at $25^{\circ} \mathrm{C}$ were easily observed (part a in Figure 1 of the Supporting Information) as a broad doublet at $6.31 \mathrm{ppm}$ in the experiment at $75{ }^{\circ} \mathrm{C}$ (part c in Figure 1 of the Supporting Information) and the same occurred with the methoxy-group signals. In the coalescence temperature, the signal of collapsed groups broadened and decreased in intensity. Figure 2 shows the minimized energy forms of the two rotamers of compound 12f, calculated by semi-empirical method PM3. ${ }^{41}$ The elimination of the bulky protecting groups led to the evanescence of the above-mentioned restricted rotation in all the compounds.

All the isopropoxy-protecting groups of dihydroisoquinolines $\mathbf{6 , 7}$, and $\mathbf{9}$ and fully aromatic systems 10-12 were removed using $\mathrm{AlCl}_{3}$ in $\mathrm{CH}_{2} \mathrm{Cl}_{2},{ }^{24-26,42}$ giving a good yield of valuable phenols 13-18. ${ }^{43,44}$ Despite the advantage of working with the protected phenol groups, the synthesis was performed without this protection in $\mathbf{4}$, as demonstrated with the synthesis of $17 \mathbf{c}$ and $\mathbf{1 5 a}$. Lamellarin analogs 1318 were obtained as reddish oils or white solids and their structures were confirmed by ${ }^{1} \mathrm{H}-$ and ${ }^{13} \mathrm{C}$ NMR, using heteronuclear bi-dimensional correlations such as HSQC, HMBC, and also MS and HRMS.

\section{Biological results}

A panel of three human tumor cell lines was used to evaluate the cytotoxic potential of the Lam-D analogs: A-549 lung carcinoma NSCL, HT-29 colon carcinoma cells, and MDA-MB-231 breast adenocarcinoma.

A conventional colorimetric assay was set up to estimate $\mathrm{GI}_{50}$ values, i.e. the drug concentration which causes $50 \%$ of cell growth inhibition after 72 hours of continuous exposure to the test molecule. Lam-D was included in the test for comparison purposes. The results obtained are shown in Table 2.

More than $75 \%$ of the open-chain Lam-D analogs tested showed cytotoxicity in a low micromolar $\mathrm{GI}_{50}$ range. Molecular simplification of Lam-D by removing the lactone ring from all the analogs and by 
additional elimination of one aryl group in derivatives $\mathbf{1 3}$ and $\mathbf{1 6}$ produced a decrease in activity with respect to Lam-D. However, interestingly these data provide crucial information about the importance of the full structure for the biological activity of the molecules in spite of their low solubility in the biological medium. In a general overview, oxidized derivatives showed greater activity than the corresponding reduced analogs. ${ }^{13}$ Derivatives with electron-withdrawing substituents, such as nitrogroups (i.e. 14m and $\mathbf{1 7} \mathbf{m}$ ) decreased activity, and this decrease was dramatic with the introduction of a $\mathrm{OCF}_{3}$ substituent such as in $\mathbf{1 4 i}$ and $\mathbf{1 7}$. The substitution pattern given by electron donor groups, such as $\mathrm{OiPr}, \mathrm{NMe}_{2}, \mathrm{OMe}$ and $\mathrm{OH}$, was fundamental for activity. Comparison of $\mathbf{6 c}$ and $\mathbf{6 d}$ shows the importance of the free $p$-phenol on the aryl on position 1 of the scaffold. Although few $O$-protected phenol analogs, such as $\mathbf{6 c}, \mathbf{7 a}, \mathbf{7 c}$, and 7f, presented cytotoxic activity, an important gain in activity was displayed by the same compounds with free $\mathrm{OH}$ functions. This observation can probably be attributed to the additional capacity of these analogs to perform hydrogen bonds with the active sites, as described for Lam-D. ${ }^{13}$ Although in the present work it has not been demonstrated the binding of this analogs with the same DNA-topI complex, other factors that could increase the activity are the solubility or the membrane-crossing issues. The donor effect of the methoxy-substituents may explain why $14 \mathrm{~g}$ and $17 \mathrm{~g}$ were quite active, even without the possibility of acting as hydrogen bond donors. Compounds 18a, 17c, 18e, 18d and Lam-D had identical substituents on the scaffold and on the aryl of position 1 , and proven a gradation in activity potency with the increase on the substitution of the aryl on position 2 of scaffold, despite 18e inactivity, presumably due to a lack of planarity probably by sterical hindrance. The simplified analog 17c maintained 63\% of activity of Lam-D in HT29 cells and most of this behaviour remained in the C4"-OH (same position as C-20 in Lam-D) group, as shown by 18a. To our knowledge, the open lactone compound 18d may produce lactonization in a physiological environment. Therefore, 18d must be considered for further study as a possible pharmacodynamic improvement for the validated Lam-D lead.

\section{Conclusion}


Here we performed a SAR study using the marine alkaloid Lam-D. Efficient and convergent modular synthetic protocols were applied in the "diverted total" synthesis of more than 40 analogs of the natural product. This strategy allowed the introduction of structural elements that have not been studied previously in the lamellarin series. Thus, the SAR information provided in this study expands our knowledge about these compounds beyond substitutions on the core structure, which has already been provided by other groups.

Overall, our results are consistent with previous findings, such as the critical importance for cytotoxic activity of the planarity of the tricyclic isoquinoline motif. In addition, compounds with $\mathrm{OH}$ hydrogen bond donors at C-8 and C-4" were generally more potent than other analogs. Not surprisingly, compound 18d, which showed most resemblance to Lam-D, was the most potent compound against the three cell lines tested. This observation may be due to partial lactonization to give Lam-D under the assay conditions.

However, remarkable retention of activity was observed for monoaryl analogs $\mathbf{1 3 c}$ and $\mathbf{1 6 c}$ against HT29 colon carcinoma cells, toward which these compounds were only ca. 5-fold less potent than Lam-D. Furthermore, the moderate activity of compound 17n against the A-549 and MDA-MB-231 cell lines (low micromolar) indicates that heterocyclic motifs may be included in a second-generation library. However, the hydrogen bond donor at C-20 should be preserved in future library designs. On the basis of this work it is clear the importance of an extensive bioprospection of the natural sources to find lead candidates more than constructing ponderous libraries.

\section{Experimental Section}

A) General Procedures for Cross-Coupling Reactions. Synthesis of Monoaryl-derivatives 6 A

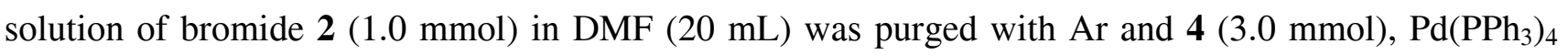
(0.1 mmol), and $2 \mathrm{M} \mathrm{K}_{2} \mathrm{CO}_{3}(3.0 \mathrm{mmol})$ were added. The reaction mixture was stirred at $125^{\circ} \mathrm{C}$ and followed by TLC until absence of starting material. The solvent was removed after cooling to room 
temperature and the residue was dissolved in EtOAc. The organic solution was washed with water and brine, dried and concentrated to give a crude material, which was later purified by column chromatography on silica gel. Elution with hexane/EtOAc (90:10 to 75:25) gave 6 (yield 32-92\%).

\section{B) General Procedures for Cross-Coupling Reactions. Synthesis of Diaryl-derivatives 7 A solution}

of 1,2-dibromide 3 (189 mg, $0.4 \mathrm{mmol})$ in DMF (8 mL) was purged with Ar for $10 \mathrm{~min}$ and 4 (2.4 mmol), $\mathrm{Pd}\left(\mathrm{PPh}_{3}\right)_{4}(46 \mathrm{mg}, 0.04 \mathrm{mmol})$, and $2 \mathrm{M} \mathrm{K}_{2} \mathrm{CO}_{3}(2.4 \mathrm{mmol})$ were added. The reaction mixture was stirred at $125^{\circ} \mathrm{C}$ and was then subjected to HPLC until disappearance of starting material or a maximum $20 \mathrm{~h}$. The solvent was removed after cooling to room temperature and the residue was dissolved in EtOAc. The organic solution was washed with water and brine, dried and concentrated to give a crude material, which was later purified by column chromatography on silica gel. Elution with hexane/EtOAc (75:25 to 40:60) gave 7 (yield 34-87\%).

C) General Procedure for Regioselective Bromination of 6. NBS (1.20 mmol) was added in one portion to a solution of $6(1.00 \mathrm{mmol})$ in THF $(13 \mathrm{~mL})$. The mixture was stirred at $70{ }^{\circ} \mathrm{C}$ under $\mathrm{Ar}$ for 90 min. The solvent was removed and the residue was purified by flash chromatography. Elution with hexane/AcOEt (90:10 to 70:30) gave $\mathbf{8}$ (yield $84 \%$ - quantitative).

\section{D) General Procedures for Cross-Coupling Reactions. Synthesis of Diaryl-derivatives 9a-c} Arylboronic acids $4(3.0 \mathrm{mmol}), \mathrm{Pd}\left(\mathrm{PPh}_{3}\right)_{4}(0.1 \mathrm{mmol})$, and $2 \mathrm{M} \mathrm{K}_{2} \mathrm{CO}_{3}(3.0 \mathrm{mmol})$ were added to a purged solution of bromide $8(1.0 \mathrm{mmol})$ in DMF $(20 \mathrm{~mL})$. The reaction mixture was stirred at $125^{\circ} \mathrm{C}$ for the time indicated for each compound (see Supporting Information). The solvent was removed and the residue was dissolved in EtOAc. The organic solution was washed with water and brine, dried and concentrated to give a crude material, which was later purified by column chromatography on silica gel. Elution with hexane/EtOAc (75:25 to 40:60) gave 9a-c (yield 71-89\%).

E) General Procedures for Cross-Coupling Reactions. Synthesis of diaryl-derivatives 9d-i. A solution of bromide $8(1.0 \mathrm{eq})$ in DMF $(20 \mathrm{~mL})$ was purged with $\mathrm{Ar}$ for $10 \mathrm{~min}$ and pinacol 
phenylboronate $5(1.0 \mathrm{mmol}), \mathrm{Pd}\left(\mathrm{PPh}_{3}\right)_{4}(10 \%)$, and $2 \mathrm{M} \mathrm{K}_{3} \mathrm{PO}_{4}(3.0 \mathrm{mmol})$ were added. The reaction mixture was stirred at $115^{\circ} \mathrm{C}$ and another portion of boronate $(2.0 \mathrm{mmol})$ was added drop-wise using a syringe pump during the first hour of reaction. The solvent was removed and the residue was dissolved in EtOAc. The organic solution was washed with water and brine, dried and concentrated to give a crude material, which was later purified by column chromatography on silica gel. Elution with hexane/EtOAc (75:25 to 60:40) gave 9d-i (yield 81\%-quant).

F) General Procedure for Oxidation. Synthesis of compounds 10- 12. A mixture of 6, 7 or 9 (1.0 mmol) and DDQ $(1.3 \mathrm{mmol})$ in dry $\mathrm{CHCl}_{3}(15 \mathrm{~mL})$ was purged with $\mathrm{Ar}$ in a sealed vessel and microwaved at $120^{\circ} \mathrm{C}$ for $10 \mathrm{~min}$. The organic solution was washed with $2 \mathrm{M} \mathrm{NaOH}$, water, and brine and then dried $\left(\mathrm{MgSO}_{4}\right)$, filtered and evaporated in vacuum. Washing with $\mathrm{NaOH}$ was avoided for products with free phenolic groups. Purification by column chromatography on silica gel eluting with hexane/AcOEt (85:15 to 60:40) gave 10-12 (yield 48-95\%).

G) General Method for Deprotection. Preparation of compounds 13-18. Anhydrous $\mathrm{AlCl}_{3}(1.3$ $\mathrm{mmol}$ ) for each isopropoxy ether was added to a solution of compound $\mathbf{6}, \mathbf{7}$, or 9-12 (1 mmol) in dry $\mathrm{CH}_{2} \mathrm{Cl}_{2}(1 \mathrm{~mL})$. The mixture was sonicated for $10 \mathrm{~min}$, quenched with sat. $\mathrm{NH}_{4} \mathrm{Cl}$, then washed with water, and with brine. The aqueous solution was extracted with AcOEt. The organic extracts were dried and evaporated. The crude was purified by flash chromatography to give the entitled compounds (yield $30-96 \%)$.

Methyl 8-hydroxy-1-(4-hydroxy-3-methoxyphenyl)-9-methoxy-5,6-dihydropyrrolo[2,1-a] isoquinoline-3-carboxylate (13c). Following the general procedure $\mathrm{G}$ and starting from $\mathbf{6 c}$ (48 $\mathrm{mg}$, $0.11 \mathrm{mmol}$ ), elution with hexane/ AcOEt (80:20 to 70:30) gave a white solid (19 mg, 44\%). M. p. $(\mathrm{MeCN}) \quad 205-207^{\circ} \mathrm{C}$. IR (film) v 3424, 1696, 1439, $1246 \mathrm{~cm}^{-1} .{ }^{1} \mathrm{H} \mathrm{NMR}\left(\mathrm{CDCl}_{3}, 400 \mathrm{MHz}\right) \delta 2.98(\mathrm{t}, J$ $=6.4 \mathrm{~Hz}, 2 \mathrm{H}, \mathrm{H6}) ; 3.47(\mathrm{~s}, 3 \mathrm{H}, \mathrm{OMe}) ; 3.84(\mathrm{~s}, 3 \mathrm{H}, \mathrm{OMe}) ; 3.86(\mathrm{~s}, 3 \mathrm{H}, \mathrm{OMe}) ; 4.59(\mathrm{t}, J=6.4 \mathrm{~Hz}, 2 \mathrm{H}$, $\mathrm{H} 5) ; 5.62(\mathrm{~s}, 1 \mathrm{H}, \mathrm{OH}) ; 5.63(\mathrm{~s}, 1 \mathrm{H}, \mathrm{OH}) ; 6.78(\mathrm{~s}, 1 \mathrm{H}) ; 6.85(\mathrm{~s}, 1 \mathrm{H}) ; 6.91-6.97(\mathrm{~m}, 4 \mathrm{H}) .{ }^{13} \mathrm{C} \mathrm{NMR}$ $\left(\mathrm{CDCl}_{3}, 100 \mathrm{MHz}\right) \delta 28.9(\mathrm{t}) ; 42.5(\mathrm{t}) ; 51.1(\mathrm{q}) ; 55.6(\mathrm{q}) ; 56.0(\mathrm{q}) ; 108.1(\mathrm{~d}) ; 112.0(\mathrm{~d}) ; 113.8(\mathrm{~d}) ; 114.3$ 
(d); 119.1 (d); 120.0 (s); 120.5 (s); 121.5 (s); 122.4 (d); 126.8 (s); 128.6 (s); 131.7 (s); 144.5 (s); 144.9 (s); 145.0 (s); 146.4 (s); 161.7 (s). MS (MALDI-TOF) m/z 395 (M, 100); 396 (M+1, 26). HRMS m/z calcd. for $\mathrm{C}_{22} \mathrm{H}_{21} \mathrm{NO}_{6} 395.1369$, found 395.1366.

\section{Methyl 1-(2,5-dimethoxyphenyl)-8-hydroxy-9-methoxy-5,6-dihydropyrrolo[2,1-a]isoquinoline-3-}

carboxylate (13e). Following the general procedure $\mathrm{G}$ and starting from $\mathbf{6 e}(17 \mathrm{mg}, 0.04 \mathrm{mmol})$, elution with hexane/ AcOEt (80:20 to 70:30) gave a white solid (12 mg, 76\%). M. p. (MeCN) 96-100 ${ }^{\circ} \mathrm{C}$. IR (film) v 3417, 1697, $1244 \mathrm{~cm}^{-1} .{ }^{1} \mathrm{H} \mathrm{NMR}\left(\mathrm{CDCl}_{3}, 400 \mathrm{MHz}\right) \delta 2.99(\mathrm{t}, J=6.8 \mathrm{~Hz}, 2 \mathrm{H}, \mathrm{H} 6) ; 3.42$ (s, 3H, OMe); 3.65 (s, 3H, OMe); 3.75 (s, 3H, OMe); 3.83 (s, 3H, OMe); $4.62(\mathrm{t}, J=6.8 \mathrm{~Hz}, 2 \mathrm{H}, \mathrm{H} 5) ; 5.55$ $(\mathrm{s}, 1 \mathrm{H}, \mathrm{OH}) ; 6.67(\mathrm{~s}, 1 \mathrm{H}) ; 6.75(\mathrm{~s}, 1 \mathrm{H}) ; 6.85\left(\mathrm{dd}, J=8.7,2.8 \mathrm{~Hz}, 1 \mathrm{H}, \mathrm{H} 4^{\prime}\right) ; 6.89$ (d, $J=8.7 \mathrm{~Hz}, 1 \mathrm{H}$, H3'); $6.90\left(\mathrm{~d}, J=2.8 \mathrm{~Hz}, 1 \mathrm{H}, \mathrm{H6}{ }^{\prime}\right) ; 7.02(\mathrm{~s}, 1 \mathrm{H}) .{ }^{13} \mathrm{C} \mathrm{NMR}\left(\mathrm{CDCl}_{3}, 100 \mathrm{MHz}\right) \delta 28.7(\mathrm{t}) ; 42.5(\mathrm{t}) ; 51.0$ (q); $55.4(\mathrm{q}) ; 55.8(\mathrm{q}) ; 56.2(\mathrm{q}) ; 107.7$ (d); $112.1(\mathrm{~d}) ; 113.5$ (d); $113.6(\mathrm{~d}) ; 116.7(\mathrm{~s}) ; 117.4$ (d); 119.9 (d); 120.1 (s); 121.0 (s); 126.3 (s); 126.5 (s); 132.7 (s); 144.8 (s); 145.0 (s); 151.6 (s); 153.6 (s); 161.8 (s). MS (MALDI-TOF) m/z 409 (M, 100); 410 (M+1, 43). ; MS (ESI-TOF) m/z 410 (M+1, 100). HRMS $m / z$ calcd. for $\mathrm{C}_{23} \mathrm{H}_{24} \mathrm{NO}_{6} 410.1598$, found 410.1598 .

\section{Methyl 1-(4-dimethylaminophenyl)-8-hydroxy-9-methoxy-5,6-dihydropyrrolo[2,1-a]isoquinoline-}

3-carboxylate (131). Following the general procedure $\mathrm{G}$ and starting from $\mathbf{6 l}(31 \mathrm{mg}, 0.07 \mathrm{mmol})$, elution with hexane/ AcOEt (80:20 to 70:30) gave a white solid (25 mg, 90\%). M. p. (MeCN) 169-170 ${ }^{\circ} \mathrm{C} . \quad$ IR (film) $v 3441,2925,1693,1439,1194 \mathrm{~cm}^{-1} .{ }^{1} \mathrm{H}$ NMR $\left(\mathrm{CDCl}_{3}, 400 \mathrm{MHz}\right) \delta \quad 2.95-2.96(\mathrm{~m}, 8 \mathrm{H}$, H6, $\mathrm{NMe}_{2}$ ); 3.47 (s, 3H, OMe); 3.83 (s, 3H, OMe); 4.57 (t, J = 6.4 Hz, 2H, H5); 5.58 (bs, 1H, OH); 6.76 $(\mathrm{s}, 1 \mathrm{H}) ; 6.77(\mathrm{~d}, J=8.8 \mathrm{~Hz}, 2 \mathrm{H}) ; 6.90(\mathrm{~s}, 1 \mathrm{H}) ; 6.95(\mathrm{~s}, 1 \mathrm{H}) ; 7.31(\mathrm{~d}, J=8.8 \mathrm{~Hz}, 2 \mathrm{H}) .{ }^{13} \mathrm{C} \mathrm{NMR}\left(\mathrm{CDCl}_{3}\right.$, $100 \mathrm{MHz}) \delta 28.9(\mathrm{t}) ; 40.8(\mathrm{q}) ; 42.5(\mathrm{t}) ; 51.0(\mathrm{q}) ; 55.6(\mathrm{q}) ; 108.2(\mathrm{~d}) ; 112.8(2 \mathrm{~d}) ; 113.7$ (d); $119.2(\mathrm{~d}) ;$ 119.9 (s); 120.9 (s); 121.7 (s); 124.7 (s); 126.7 (s); 130.1 (2d); 131.7 (s); 144.8 (s); 144.9 (s); 149.6 (s); 161.8 (s). MS (MALDI-TOF) m/z 392 (M, 100). MS (ESI-TOF) $m / z 393$ (M+1, 100). HRMS m/z calcd. for $\mathrm{C}_{23} \mathrm{H}_{25} \mathrm{~N}_{2} \mathrm{O}_{4}$ 393.1809, found 393.1809. 


\section{Methyl 1,2-bis(3,5-dimethoxy-4-hydroxyphenyl)-8-hydroxy-9-methoxy-5,6-dihydropyrrolo[2,1-}

a] isoquinoline-3-carboxylate $\left(\mathbf{1 4 a}, \mathbf{R}^{4}=\mathbf{O H}\right)$. Following the general procedure $\mathrm{G}$ and starting from $\mathbf{7 a}$ (24 mg, $0.04 \mathrm{mmol})$ and an excess of $\mathrm{AlCl}_{3}(0.8 \mathrm{mmol})$, on elution with hexane/ AcOEt (60:40 to 40:60) a yellowish solid (12 mg, 58\%) was obtained. M. p. (MeCN) $118-120 \quad{ }^{\circ} \mathrm{C} . \quad$ IR (film) v 3430, 1689, 1437, $1210 \mathrm{~cm}^{-1} .{ }^{1} \mathrm{H} \mathrm{NMR}\left(\mathrm{CDCl}_{3}, 400 \mathrm{MHz}\right) \delta 3.02(\mathrm{t}, J=6.4 \mathrm{~Hz}, 2 \mathrm{H}, \mathrm{H} 6) ; 3.40$ (s, 3H, OMe); 3.64 (s, 3H, OMe); 3.68 (s, 6H, 2OMe); $3.71(\mathrm{~s}, 6 \mathrm{H}, 2 \mathrm{OMe}) ; 4.59$ (t, $J=6.4 \mathrm{~Hz}, 2 \mathrm{H}, \mathrm{H} 5)$; $5.41(\mathrm{bs}, 1 \mathrm{H}, \mathrm{OH}) ; 5.43(\mathrm{bs}, 1 \mathrm{H}, \mathrm{OH}) ; 5.58(\mathrm{bs}, 1 \mathrm{H}, \mathrm{OH}) ; 6.39(\mathrm{~s}, 2 \mathrm{H}) ; 6.40(\mathrm{~s}, 2 \mathrm{H}) ; 6.67$ (s, $1 \mathrm{H}) ; 6.78$ $(\mathrm{s}, 1 \mathrm{H}) .{ }^{13} \mathrm{C} \mathrm{NMR}\left(\mathrm{CDCl}_{3}, 100 \mathrm{MHz}\right) \delta 28.9(\mathrm{t}) ; 42.9(\mathrm{t}) ; 50.9(\mathrm{q}) ; 55.5(\mathrm{q}) ; 56.2(2 \mathrm{q}) ; 56.4(2 \mathrm{q}) ; 107.8$ (2d); 107.9 (2d); 108.3 (d); 113.7 (d); 117.8 (s); 120.2 (s); 121.4 (s); 126.3 (s); 126.5 (s); 126.9 (s); 131.2 (s); 132.7 (s); 133.3 (s); 133.5 (s); 144.9 (s); 146.0 (2s); 146.9 (2s); 162.4 (s). MS (MALDI-TOF) $m / z 577(\mathrm{M}, 100) ; 578(\mathrm{M}+1,40)$. HRMS $m / z$ calcd. for $\mathrm{C}_{31} \mathrm{H}_{31} \mathrm{NO}_{10} 577.1948$, found 577.1942.

\section{Methyl 8-hydroxy-1,2-bis(4-hydroxy-3-methoxyphenyl)-9-methoxy-5,6-dihydropyrrolo[2,1-} a]isoquinoline-3-carboxylate (14c). Following the general procedure $\mathrm{G}$ and starting from $\mathbf{7 c}(28 \mathrm{mg}$, $0.05 \mathrm{mmol}$ ), elution with hexane/ AcOEt (50:50 to AcOEt) gave a yellowish solid (15 mg, 60\%). M. p. $(\mathrm{MeCN}) \quad 237-239{ }^{\circ} \mathrm{C}$. IR (film) $v 3423,1688,1438,1235,1199 \mathrm{~cm}^{-1} .{ }^{1} \mathrm{H} \mathrm{NMR}\left(\mathrm{CDCl}_{3}, 400 \mathrm{MHz}\right) \delta$ $3.01(\mathrm{t}, J=6.4 \mathrm{~Hz}, 2 \mathrm{H}, \mathrm{H} 6) ; 3.38(\mathrm{~s}, 3 \mathrm{H}, \mathrm{OMe}) ; 3.62(\mathrm{~s}, 3 \mathrm{H}, \mathrm{OMe}) ; 3.63$ (s, 3H, OMe); 3.65 (s, 3H, $\mathrm{OMe}$ ); 4.59 (t, $J=6.4 \mathrm{~Hz}, 2 \mathrm{H}, \mathrm{H} 5) ; 5.50$ (bs, 1H, OH); 5.53 (bs, 1H, OH); 5.59 (bs, 1H, OH); 6.55 and $6.58(2 \mathrm{~d}, J=1.6 \mathrm{~Hz}, 2 \mathrm{H}, \mathrm{H} 2$ ', H2”); 6.63 (s, 1H); 6.70 and 6.75 (2dd, $J=8.4,1.6 \mathrm{~Hz}, 2 \mathrm{H}, \mathrm{H} 6$ ', H6”); $6.77(\mathrm{~s}, 1 \mathrm{H}) ; 6.78$ and $6.83(2 \mathrm{~d}, J=8.4 \mathrm{~Hz}, 2 \mathrm{H}, \mathrm{H} 5, \mathrm{H} 5 ”) .{ }^{13} \mathrm{C} \mathrm{NMR}\left(\mathrm{CDCl}_{3}, 100 \mathrm{MHz}\right): \delta 28.9(\mathrm{t})$; 42.9 (t); 50.8 (q); 55.4 (q); 55.8 (q); 55.9 (q); 108.2 (d); 113.3 (d); 113.5 (d); 113.6 (d); 113.9 (d); 114.1 (d); 117.8 (s); 120.4 (s); 121.4 (s); 123.9 (d); 124.3 (d); 126.9 (s); 127.3 (s); 127.5 (s); 131.3 (s); 132.7 (s); 144.0 (s); 144.3 (s); 144.9 (s); 145.4 (2s); 146.3 (s); 162.5 (s). MS (MALDI-TOF) 517 (M, 100); $518(\mathrm{M}+1,15)$. HRMS $m / z$ calcd. for $\mathrm{C}_{29} \mathrm{H}_{27} \mathrm{NO}_{8} 517.1737$, found 517.1731.

Methyl 1,2-bis(3,4-dimethoxyphenyl)-8-hydroxy-9-methoxy-5,6-dihydropyrrolo[2,1a]isoquinoline-3-carboxylate (14f). Following the general procedure $\mathrm{G}$ and starting from $\mathbf{7 f}(92.0 \mathrm{mg}$, 
$0.16 \mathrm{mmol}$ ), elution with hexane/ AcOEt (60:40 to 40:60) gave $\mathbf{1 4 f}$ as a reddish oil (50.9 mg, 60\%). IR (film) v 3410, 1691, 1437, $1254 \mathrm{~cm}^{-1} .{ }^{1} \mathrm{H} \mathrm{NMR}\left(\mathrm{CDCl}_{3}, 400 \mathrm{MHz}\right) \delta 3.01(\mathrm{t}, J=6.5 \mathrm{~Hz}, 2 \mathrm{H}, \mathrm{H} 6) ; 3.36$ (s, 3H, OMe); 3.62 (s, 3H, OMe); 3.63 (s, 3H, $\left.\mathrm{CO}_{2} \mathrm{Me}\right) ; 3.67$ (s, 3H, OMe); 3.83 (s, 3H, OMe); 3.84 (s, $3 \mathrm{H}, \mathrm{OMe}) ; 4.59$ (t, $J=6.5 \mathrm{~Hz}, 2 \mathrm{H}, \mathrm{H} 5) ; 5.67(\mathrm{bs}, 1 \mathrm{H}, \mathrm{OH}) ; 6.62(\mathrm{~d}, J=1.6 \mathrm{~Hz}, 1 \mathrm{H}) ; 6.64(\mathrm{~d}, J=1.6 \mathrm{~Hz}$, $1 \mathrm{H}) ; 6.66(\mathrm{~s}, 1 \mathrm{H}) ; 6.71(\mathrm{dd}, J=8.4$ and $1.6 \mathrm{~Hz}, 1 \mathrm{H}) ; 6.72(\mathrm{~s} 1 \mathrm{H}) ; 6.74(\mathrm{dd}, J=8.4$ and $1.6 \mathrm{~Hz}, 1 \mathrm{H})$; $6.76(\mathrm{~d}, J=8.4 \mathrm{~Hz}, 1 \mathrm{H}) ; 6.77(\mathrm{~d}, J=8.4 \mathrm{~Hz}, 1 \mathrm{H}) .{ }^{13} \mathrm{C} \mathrm{NMR}\left(\mathrm{CDCl}_{3}, 100 \mathrm{MHz}\right): \delta 28.8(\mathrm{t}) ; 42.8(\mathrm{t}) ;$ 50.8 (q); 55.3 (q); 55.5 (q); 55.6 (q); 55.7 (q); 55.8 (q); $108.2(\mathrm{~d}) ; 109.9$ (d); 110.9 (d); 113.7 (d); 114.1 (d); 114.3 (d); 117.7 (s); 120.2 (s); 122.9 (d); 123.4 (d); 123.4 (s); 126.8 (s); 127.8 (s); 128.0 (s); 131.2 (s); 132.5 (s); 141.6 (s); 144.8 (s); 147.3 (s); 147.5 (s); 147.5 (s); 148.5 (s); 162.3 (s). MS (MALDITOF) $m / z, 545$ (M, 100). HRMS $m / z$ calcd. for $\mathrm{C}_{31} \mathrm{H}_{31} \mathrm{NO}_{8}$ 545.2050, found 545.2044.

\section{Methyl 8-hydroxy-9-methoxy-1,2-bis(4-methoxyphenyl)-5,6-dihydropyrrolo[2,1-a]isoquinoline-3-}

carboxylate (14g). Following the general procedure $\mathrm{G}$ and starting from $\mathbf{7 g}(18.2 \mathrm{mg}, 0.034 \mathrm{mmol})$, elution with hexane/ AcOEt (80:20 to 50:50) gave $\mathbf{1 4 g}(6.8 \mathrm{mg}, 41 \%)$ as a reddish oil. IR (film) $v 2931,1697 \mathrm{~cm}^{-1} .{ }^{1} \mathrm{H} \mathrm{NMR}\left(\mathrm{CDCl}_{3}, 400 \mathrm{MHz}\right) \delta 3.01(\mathrm{t}, J=6.5 \mathrm{~Hz}, 2 \mathrm{H}, \mathrm{H} 6) ; 3.34$ (s, 3H, OMe); 3.59 (s, 3H, $\left.\mathrm{CO}_{2} \mathrm{Me}\right) ; 3.76$ (s, 3H, OMe); 3.77 (s, 3H, OMe); 4.59 (t, J = 6.5 Hz, 2H, H5); 5.52 (bs, 1H, $\mathrm{OH}) ; 6.50(\mathrm{~s}, 1 \mathrm{H}) ; 6.74(\mathrm{~d}, J=9.0 \mathrm{~Hz}, 2 \mathrm{H}) ; 6.76(\mathrm{~s}, 1 \mathrm{H}) ; 6.79(\mathrm{~d}, J=8.6 \mathrm{~Hz}, 2 \mathrm{H}) ; 7.03(\mathrm{~d}, J=9.0 \mathrm{~Hz}$, $2 \mathrm{H}) ; 7.06(\mathrm{~d}, J=8.6 \mathrm{~Hz}, 2 \mathrm{H}) .{ }^{13} \mathrm{C} \mathrm{NMR}\left(\mathrm{CDCl}_{3}, 100 \mathrm{MHz}\right) \delta 28.9(\mathrm{t}) ; 42.9(\mathrm{t}) ; 50.7(\mathrm{q}) ; 55.0(\mathrm{q}) ; 55.2$ (q); 55.2 (q); 108.2 (d); 109.7 (d); 112.5 (2d); $113.6(2 d) ; 117.9$ (s); 120.5 (s); 121.2 (s); 126.8 (s); 127.6 (s); $127.8(\mathrm{~s}) ; 131.4$ (s); 131.6 (2d); 132.3 (2d); 132.6 (s); 144.7 (s); 144.8 (s); 157.9 (s); 158.2 (s); 162.5 (s). MS (MALDI-TOF) $m / z 485$ (M). HRMS $m / z$ calcd. for $\mathrm{C}_{29} \mathrm{H}_{27} \mathrm{NO}_{6} 485.1838$, found 485.1833.

\section{Methyl 8-hydroxy-9-methoxy-1,2-bis(4-trifluoromethoxyphenyl)-5,6-dihydropyrrolo[2,1-} a]isoquinoline-3-carboxylate (14i). Following the general procedure $\mathrm{G}$ and starting from $7 \mathbf{i}$ (25.9 $\mathrm{mg}$, $0.041 \mathrm{mmol})$, elution with hexane/ AcOEt (85:15 to 65:35) gave 14i (18.0 mg, 75\%) as a reddish oil. IR (film) v 2927, $1699 \mathrm{~cm}^{-1} \cdot{ }^{1} \mathrm{H} \mathrm{NMR}\left(\mathrm{CDCl}_{3}, 400 \mathrm{MHz}\right) \delta 3.02(\mathrm{t}, J=6.4 \mathrm{~Hz}, 2 \mathrm{H}, \mathrm{H} 6) ; 3.31(\mathrm{~s}, 3 \mathrm{H}$, $\mathrm{OMe}) ; 3.58$ (s, 3H, $\left.\mathrm{CO}_{2} \mathrm{Me}\right) ; 4.61(\mathrm{t}, J=6.4 \mathrm{~Hz}, 2 \mathrm{H}, \mathrm{H} 5) ; 5.57$ (bs, 1H, OH); $6.33(\mathrm{~s}, 1 \mathrm{H}) ; 6.79(\mathrm{~s}, 1 \mathrm{H})$; 
7.04-7.06 (m, 2H); 7.10-7.17 (m, 6H). ${ }^{13} \mathrm{C} \mathrm{NMR}\left(\mathrm{CDCl}_{3}, 100 \mathrm{MHz}\right) \delta 28.8(\mathrm{t}) ; 42.9(\mathrm{t}) ; 50.8(\mathrm{q}) ; 54.9$ (q); 107.9 (d); 113.8 (d); 118.2 (s); 119.5 (2d); 119.7 (q); 119.9 (q); 121.0 (s); 121.1 (2d); 121.7 (s); 127.0 (s); 131.3 (s); 131.6 (s); 131.8 (2d); 132.6 (2d); 133.8 (s); 134.3 (s), 144.9 (s); 145.1 (s); 147.8 (s); 147.9 (s); 161.9 (s). MS (MALDI-TOF) m/z 593 (M); $594(\mathrm{M}+1)$. HRMS m/z calcd. for $\mathrm{C}_{29} \mathrm{H}_{21} \mathrm{~F}_{6} \mathrm{NO}_{6}$ 593.1273, found 593.1268

Methyl 8-hydroxy-1,2-bis(4-hydroxyphenyl)-9-methoxy-5,6-dihydropyrrolo[2,1-a]isoquinoline-3carboxylate (14j). Following the general procedure $G$ and starting from $\mathbf{7 j}$ (28.6 mg, $0.049 \mathrm{mmol}$ ), elution with hexane/ AcOEt (60:40 to AcOEt) gave 14j (15.0 mg, 67\%) as a pale solid. M. p. (MeCN) 190-5 ${ }^{\circ} \mathrm{C}$. IR (film) v 3194, 1683, 1436, $1267 \mathrm{~cm}^{-1} .{ }^{1} \mathrm{H}$ NMR (DMSO-d $\left.6,400 \mathrm{MHz}\right) \delta 2.92(\mathrm{~m}, 2 \mathrm{H}$, H6); 3.21 (s, 3H, OMe); 3.48 (s, 3H, $\left.\mathrm{CO}_{2} \mathrm{Me}\right) ; 4.43$ (m, 2H, H5); 6.39 (s, 1H); 6.55 (d, J = 8.1 Hz, 2H); $6.67(\mathrm{~m}, 3 \mathrm{H}) ; 6.85(\mathrm{~d}, J=8.2 \mathrm{~Hz}, 2 \mathrm{H}) ; 6.88(\mathrm{~d}, J=8.2 \mathrm{~Hz}, 2 \mathrm{H}) ; 9.16(\mathrm{bs}, 2 \mathrm{H}, \mathrm{OH}) ; 9.31$ (bs, 1H, OH). ${ }^{13} \mathrm{C}$ NMR (DMSO-d 6 , 100 MHz): $\delta 27.8$ (t); 42.4 (t); 50.4 (q); 54.5 (q); 108.8 (d); 113.8 (2d); 114.8 (d); $115.0(2 \mathrm{~d}) ; 117.0(\mathrm{~s}) ; 118.8$ (s); 120.7 (s); 125.5 (s); 125.6 (s); 126.3 (s); 130.8 (s); 131.2 (2d); 131.9 (2d); 132.2 (s); 145.6 (s); 145.7 (s); 155.4 (s); 155.9 (s); 161.5 (s). MS (MALDI-TOF) m/z 457 (M). HRMS $m / z$ calcd. for $\mathrm{C}_{27} \mathrm{H}_{23} \mathrm{NO}_{6} 457.1525$, found. 457.1520

\section{Methyl 8-hydroxy-1,2-bis(3-hydroxyphenyl)-9-methoxy-5,6-dihydropyrrolo[2,1-a]isoquinoline-3-}

carboxylate (14k). Following the general procedure $\mathrm{G}$ and starting from $7 \mathbf{k}(18.6 \mathrm{mg}, 0.032 \mathrm{mmol})$, elution with hexane/ AcOEt (60:40 to AcOEt) gave 14k (12.3 mg, 85\%) as a white solid. M. p. (MeCN) 128-130 ${ }^{\circ} \mathrm{C}$. IR (film) v 3299, 1680, 1440, $1202 \mathrm{~cm}^{-1} .{ }^{1} \mathrm{H}$ NMR $\left(\right.$ DMSO-d $\left._{6}, 400 \mathrm{MHz}\right) \delta 2.94(\mathrm{t}, J=$ $6.5 \mathrm{~Hz}, 2 \mathrm{H}, \mathrm{H6}$ ); 3.20 (s, 3H, OMe); 3.49 (s, $3 \mathrm{H}, \mathrm{CO}_{2} \mathrm{Me}$ ); 4.43 (m, 2H, H5); 6.39 (s, 1H); 6.48-6.50 $(\mathrm{m}, 2 \mathrm{H}) ; 6.52-6.55(\mathrm{~m}, 3 \mathrm{H}) ; 6.9-6.64(\mathrm{~m}, 1 \mathrm{H}) ; 6.94-6.98(\mathrm{t}, J=8.1 \mathrm{~Hz}, 1 \mathrm{H}) ; 7.06-7.10(\mathrm{t}, J=8.4 \mathrm{~Hz}$, $1 \mathrm{H}) ; 6.68(\mathrm{~s}, 1 \mathrm{H}) ; 9.11(\mathrm{bs}, 1 \mathrm{H}, \mathrm{OH}) ; 9.17$ (bs, 1H, OH); 9.27 (bs, 1H, OH). ${ }^{13} \mathrm{C} \mathrm{NMR}\left(\mathrm{DMSO}-\mathrm{d}_{6}, 100\right.$ MHz) $\delta 27.8(\mathrm{t}) ; 42.5(\mathrm{t}) ; 50.6(\mathrm{q}) ; 54.4(\mathrm{q}) ; 108.8(\mathrm{~d}) ; 113.1(\mathrm{~d}) ; 113.6(\mathrm{~d}) ; 114.8(\mathrm{~d}) ; 117.1(\mathrm{~d}) ; 117.3$ (d); 118.5 (s); 120.5 (s); 121.0 (d); 121.6 (d); 126.3 (s); 127.7 (d); 129.1 (d); 130.5 (s); 131.6 (s); 136.2 
(s); 136.5 (s); 145.7 (s); 145.9 (s); 155.9 (s); 157.1 (s); 161.4 (s). MS (MALDI-TOF) m/z 457 (M). HRMS $m / z$ calcd. for $\mathrm{C}_{27} \mathrm{H}_{23} \mathrm{NO}_{6} 457.1525$, found 457.1520 .

Methyl

1,2-bis(4-dimethylaminophenyl)-8-hydroxy-9-methoxy-5,6-dihydropyrrolo[2,1-

a]isoquinoline-3-carboxylate (14l). Following the general procedure $\mathrm{G}$ and starting from 111 (5.4 $\mathrm{mg}$, $0.0098 \mathrm{mmol})$, elution with hexane/ AcOEt (80:20 to 50:50) gave 14l (2.3 $\mathrm{mg}, 46 \%)$ as a white solid. M. p. $(\mathrm{MeCN}) 245-247^{\circ} \mathrm{C}$. IR (film) $v \mathrm{~cm}^{-1} .{ }^{1} \mathrm{H} \mathrm{NMR}\left(\mathrm{CDCl}_{3}, 400 \mathrm{MHz}\right) \delta 2.88\left(\mathrm{~s}, 6 \mathrm{H}, \mathrm{NMe}_{2}\right) ; 2.91$ $\left(\mathrm{s}, 6 \mathrm{H}, \mathrm{NMe}_{2}\right) ; 2.99(\mathrm{t}, J=6.5 \mathrm{~Hz}, 2 \mathrm{H}, \mathrm{H} 6) ; 3.35(\mathrm{~s}, 3 \mathrm{H}, \mathrm{OMe}) ; 3.62\left(\mathrm{~s}, 3 \mathrm{H}, \mathrm{CO}_{2} \mathrm{Me}\right) ; 4.56(\mathrm{t}, J=6.5$ $\mathrm{Hz}, 2 \mathrm{H}, \mathrm{H} 5) ; 5.48(\mathrm{bs}, 1 \mathrm{H}, \mathrm{OH}) ; 6.54(\mathrm{~s}, 1 \mathrm{H}) ; 6.59(\mathrm{~d}, J=8.8 \mathrm{~Hz}, 2 \mathrm{H}) ; 6.64(\mathrm{~d}, J=8.8 \mathrm{~Hz}, 2 \mathrm{H}) ; 6.74$ $(\mathrm{s}, 1 \mathrm{H}) ; 7.01(\mathrm{~d}, J=8.8 \mathrm{~Hz}, 2 \mathrm{H}) ; 7.02(\mathrm{~d}, J=8.8 \mathrm{~Hz}, 2 \mathrm{H}) .{ }^{13} \mathrm{C} \mathrm{NMR}\left(\mathrm{CDCl}_{3}, 100 \mathrm{MHz}\right): \delta 28.9(\mathrm{t})$; $40.5(\mathrm{t}) ; 40.5(\mathrm{q}) ; 40.7$ (q); 50.7 (q); $55.2(\mathrm{q}) ; 108.3(\mathrm{~d}) ; 111.4(2 \mathrm{~d}) ; 112.8(2 \mathrm{~d}) ; 113.4$ (d); 117.6 (s); 120.8 (s); 121.7 (s); 121.9 (s); 123.9 (s); 126.7 (s); 131.2 (2d); 131.4 (s); 131.9 (2d); 144.4 (s); 144.6 (s); 146.6 (s); 149.8 (s); 161.1 (s). MS (MALDI-TOF) $\mathrm{m} / z .511(\mathrm{M}) ; 512(\mathrm{M}+1)$. HRMS $\mathrm{m} / z$ calcd. for $\mathrm{C}_{31} \mathrm{H}_{33} \mathrm{~N}_{3} \mathrm{O}_{4}$ 511.2471, found. 511.2466.

Methyl 8-hydroxy-9-methoxy-1,2-bis(3-nitrophenyl)-5,6-dihydropyrrolo[2,1-a]isoquinoline-3carboxylate (14m). Following the general procedure $\mathrm{G}$ and starting from $\mathbf{7 m}(42.2 \mathrm{mg}, 0.076 \mathrm{mmol})$, elution with hexane/ AcOEt (80:20 to 50:50) gave $\mathbf{1 4 m}(17.0 \mathrm{mg}, 44 \%)$ as a reddish solid. M. p. $(\mathrm{MeCN}) 241-243{ }^{\circ} \mathrm{C}$. IR (film) v 2926, 1701, 1540, 1439, 1350, $1227 \mathrm{~cm}^{-1} .{ }^{1} \mathrm{H} \mathrm{NMR}\left(\mathrm{CDCl}_{3}, 400 \mathrm{MHz}\right)$ $\delta 3.06(\mathrm{t}, J=6.5 \mathrm{~Hz}, 2 \mathrm{H}, \mathrm{H} 6) ; 3.29(\mathrm{~s}, 3 \mathrm{H}, \mathrm{OMe}) ; 3.59$ (s, 3H, $\left.\mathrm{CO}_{2} \mathrm{Me}\right) ; 4.66(\mathrm{t}, J=6.5 \mathrm{~Hz}, 2 \mathrm{H}, \mathrm{H} 5)$; 5.63 (bs, 1H, OH); 6.33 (s, 1H); $6.83(\mathrm{~s}, 1 \mathrm{H}) ; 7.36-7.50(\mathrm{~m}, 4 \mathrm{H}) ; 8.00-8.02(\mathrm{~m}, 2 \mathrm{H}) ; 8.05-8.08$ (m, 2H). ${ }^{13} \mathrm{C} \mathrm{NMR}\left(\mathrm{CDCl}_{3}, 100 \mathrm{MHz}\right): \delta 28.7(\mathrm{t}) ; 43.0(\mathrm{t}) ; 51.1(\mathrm{q}) ; 55.3(\mathrm{q}) ; 107.8(\mathrm{~d}) ; 114.2(\mathrm{~d}) ; 118.6(\mathrm{~s}) ;$ 118.7 (s); 121.7 (d); 121.8 (d); 125.5 (d); 125.8 (d); 127.4 (s); 128.2 (d); 129.4 (d); 130.1 (s); 131.9 (s); 136.6 (d); 136.9 (s); 137.4 (d); 145.1 (s); 145.6 (s) 147.5 (s); 148.2 (s); 161.4 (s). MS (MALDI-TOF) $m / z 515(\mathrm{M}) ; 516(\mathrm{M}+1)$. HRMS $m / z$ calcd. for $\mathrm{C}_{27} \mathrm{H}_{21} \mathrm{~N}_{3} \mathrm{O}_{8}$ 515.1329, found. 515.1323.

Methyl 2-(2,4-dihydroxy-5-methoxyphenyl)-8-hydroxy-1-(4-hydroxy-3-methoxyphenyl)-9methoxy-5,6-dihydropyrrolo[2,1-a]isoquinoline-3-carboxylate (15d). Following the general 
procedure $\mathrm{G}$ and starting from $\mathbf{9 d}(48 \mathrm{mg}, 0.07 \mathrm{mmol})$, elution with hexane/ AcOEt (60:40 to 40:60) gave a reddish oil $(26 \mathrm{mg}, 70 \%)$. IR (film) $\vee 3419,1686,1439,1246,1197 \mathrm{~cm}^{-1} .{ }^{1} \mathrm{H} \mathrm{NMR}\left(\mathrm{CDCl}_{3}, 400\right.$ MHz) $\delta$ 2.95-3.15 (m, 2H, H6); 3.40 (s, 3H, OMe); 3.54 (s, 3H, OMe); 3.63 (s, 3H, OMe); 3.71 (s, 3H, OMe); 4.15-4.25 (m, 2H, H5); 5.54 (s, 3H, 3OH); 5.63 (s, 1H, OH); 6.31 (bs, 1H); 6.53 (bs, 1H, H2'); $6.56(\mathrm{~s}, 1 \mathrm{H}) ; 6.72(\mathrm{~s}, 1 \mathrm{H}) ; 6.75-6.79(\mathrm{~m}, 2 \mathrm{H}) ; 6.82\left(\mathrm{~d}, J=8.0 \mathrm{~Hz}, 1 \mathrm{H}, \mathrm{H} 5{ }^{\prime}\right) .{ }^{13} \mathrm{C} \mathrm{NMR}\left(\mathrm{CDCl}_{3}, 100\right.$ MHz) $\delta 28.8(\mathrm{t}) ; 43.2(\mathrm{t}) ; 51.5(\mathrm{q}) ; 55.5(\mathrm{q}) ; 55.9(\mathrm{q}) ; 56.4(\mathrm{q}) ; 102.8(\mathrm{~d}) ; 108.5(\mathrm{~d}) ; 113.4(\mathrm{~d}) ; 113.8$ (d); 114.0 (d); 114.3 (d); 118.9 (s); 120.0 (s); 122.5 (s); 123.8 (d); 126.8 (s); 126.9 (2s); 127.3 (s); 132.4 (s); 140.2 (s); 144.4 (s); 145.0 (s); 145.2 (s); 145.7 (s); 146.4 (s); 148.7 (s); 162.4 (s). MS (MALDITOF) $533(\mathrm{M}, 100) ; 534(\mathrm{M}+1,70) ; 535(\mathrm{M}+2,32)$. HRMS $m / z$ calcd. for $\mathrm{C}_{29} \mathrm{H}_{27} \mathrm{NO}_{9}$ 533.1686, found 533.1680.

Methyl 2-(2,5-dimethoxy-4-hydroxyphenyl)-8-hydroxy-1-(4-hydroxy-3-methoxyphenyl)-9methoxy-5,6-dihydropyrrolo[2,1-a]isoquinoline-3-carboxylate (15e). Following the general procedure $\mathrm{G}$ and starting from 9e $(67 \mathrm{mg}, 0.10 \mathrm{mmol})$, elution with hexane/ AcOEt (60:40 to 40:60) gave a brown solid (24 mg, 45\%). M. p. (MeCN) 140-145 ${ }^{\circ} \mathrm{C}$. IR (film) $v 3423,1688,1265,1196 \mathrm{~cm}^{-}$ 1. ${ }^{1} \mathrm{H}$ NMR $\left(\mathrm{CDCl}_{3}, 400 \mathrm{MHz}\right) \delta 3.02$ (bs, 2H, H6); 3.38 (s, 3H, OMe); 3.57 (s, 3H, OMe); 3.60 (s, 6H, 2OMe); 3.63 (s, 3H, OMe); 4.57 (bs, 2H, H5); 5.53 (s, 1H, OH); 5.57 (s, 1H, OH); 5.59 (s, 1H, OH); $6.50(\mathrm{~s}, 2 \mathrm{H}) ; 6.59(\mathrm{~s}, 1 \mathrm{H}) ; 6.66(\mathrm{~s}, 1 \mathrm{H}) ; 6.74-6.79(\mathrm{~m}, 2 \mathrm{H}) ; 6.81\left(\mathrm{~d}, J=8.0 \mathrm{~Hz}, 1 \mathrm{H}, \mathrm{H} 5{ }^{\prime}\right) .{ }^{13} \mathrm{C}$ NMR $\left(\mathrm{CDCl}_{3}, 100 \mathrm{MHz}\right) \delta 28.8(\mathrm{t}) ; 42.8(\mathrm{t}) ; 50.8(\mathrm{q}) ; 55.4(\mathrm{q}) ; 55.9(\mathrm{q}) ; 56.2(\mathrm{q}) ; 56.5(\mathrm{q}) ; 98.8(\mathrm{~d}) ; 108.3(\mathrm{~d}) ;$ 113.5 (d); 113.7 (d); 113.9 (d); 115.1 (d); 115.6 (s); 118.8 (s); 120.6 (s); 121.4 (s); 123.9 (d); 126.7 (s); 127.8 (s); 128.0 (s); 130.5 (s); 131.1 (s); 135.0 (s); 139.6 (s); 144.1 (s); 144.9 (s); 146.1 (s); 151.9 (s); 162.5 (s). MS (MALDI-TOF) 547 (M, 100); $548(\mathrm{M}+1,30)$. HRMS m/z calcd. for $\mathrm{C}_{30} \mathrm{H}_{29} \mathrm{NO}_{9}$ 547.1842, found 547.1837.

Methyl 1-(2,5-dimethoxyphenyl)-8-hydroxy-9-methoxy-2-(2,4,5-trihydroxyphenyl)-5,6dihydropyrrolo[2,1-a]isoquinoline-3-carboxylate $(\mathbf{1 5 g})$. Following the general procedure $G$ and starting from $9 \mathrm{~g}$ (22 mg, $0.03 \mathrm{mmol})$, elution with hexane/ AcOEt (60:40 to 40:60) gave a reddish oil (7 
$\mathrm{mg}, 42 \%) . \quad \mathrm{IR}($ film $) \vee 3425,1697,1465,1243 \mathrm{~cm}^{-1} .{ }^{1} \mathrm{H} \mathrm{NMR}\left(\mathrm{CDCl}_{3}, 400 \mathrm{MHz}\right) \delta 2.99(\mathrm{t}, J=6.4 \mathrm{~Hz}$, 2H, H6); 3.41 (s, 3H, OMe); 3.65 (s, 3H, OMe); 3.75 (s, 3H, OMe); 3.83 (s, 3H, OMe); 4.61 (t, J = 6.4 $\mathrm{Hz}, 2 \mathrm{H}, \mathrm{H} 5) ; 5.12$ (bs, 1H, OH); 5.34 (bs, 1H, OH); 5.54 (bs, 1H, OH); 5.78 (bs, 1H, OH); 6.67 (s, 1H); $6.75(\mathrm{~s}, 1 \mathrm{H}) ; 6.84\left(\mathrm{dd}, J=8.9,2.8 \mathrm{~Hz}, 1 \mathrm{H}, \mathrm{H} 4\right.$ ') $6.88(\mathrm{~s}, 1 \mathrm{H}) ; 6.89-6.92(\mathrm{~m}, 2 \mathrm{H}) ; 7.02(\mathrm{~s}, 1 \mathrm{H}) .{ }^{13} \mathrm{C}$ $\operatorname{NMR}\left(\mathrm{CDCl}_{3}, 100 \mathrm{MHz}\right) \delta 30.9(\mathrm{t}) ; 42.5(\mathrm{t}) ; 51.0(\mathrm{q}) ; 55.4(\mathrm{q}) ; 55.8(\mathrm{q}) ; 56.2(\mathrm{q}) ; 107.6(\mathrm{~d}) ; 112.2(\mathrm{~d}) ;$ 113.5 (d); 113.6 (d); 116.7 (s); 117.4 (d); 119.9 (d); 120.1 (s); 121.1 (s); 126.3 (d); 126.5 (s); 132.7 (s); 144.8 (s); 145.0 (s); 149.8 (s); 150.3 (s); 151.6 (s); 153.6 (s); 161.8 (s). MS (MALDI-TOF) 533 (M, 100). HRMS $m / z$ calcd. for $\mathrm{C}_{29} \mathrm{H}_{27} \mathrm{NO}_{9}$ 533.1686, found 533.1684 .

\section{Methyl 8-hydroxy-2-(2-hydroxy-4,5-dimethoxyphenyl)-1-(3-hydroxyphenyl)-9-methoxy-5,6-}

dihydropyrrolo[2,1-a]isoquinoline-3-carboxylate (15i). Following the general procedure $G$ and starting from 9i (23 mg, $0.04 \mathrm{mmol}$ ), elution with hexane/ AcOEt (60:40 to 40:60) gave a reddish oil (16 mg, 85\%). IR (film) v 3405, 1684, 1437, $1196 \mathrm{~cm}^{-1} .{ }^{1} \mathrm{H} \mathrm{NMR}\left(\mathrm{CDCl}_{3}, 400 \mathrm{MHz}\right) \delta 3.01(\mathrm{t}, J=6.8$ $\mathrm{Hz}, 2 \mathrm{H}, \mathrm{H6}$ ); 3.36 (s, 3H, OMe); 3.60 (s, 3H, OMe); 3.81 (s, 3H, OMe); 3.85 (s, 3H, OMe); 4.46-4.71 (m, 2H, H5); 4.93 (bs, 1H, OH); 5.55 (bs, 1H, OH); 5.74 (bs, 1H, OH); 6.30 (d, J= 8.6 Hz, 1H); 6.53 (s, $1 \mathrm{H}) ; 6.60(\mathrm{~d}, J=8.6 \mathrm{~Hz}, 1 \mathrm{H}) ; 6.64-6.68(\mathrm{~m}, 2 \mathrm{H}) ; 6.76(\mathrm{~s}, 1 \mathrm{H}) ; 6.78(\mathrm{~d}, J=7.2 \mathrm{~Hz}, 1 \mathrm{H}) ; 7.11(\mathrm{t}, J=7.2$ $\left.\mathrm{Hz}, 1 \mathrm{H}, \mathrm{H} 5{ }^{\prime}\right) .{ }^{13} \mathrm{C} \mathrm{NMR}\left(\mathrm{CDCl}_{3}, 100 \mathrm{MHz}\right) \delta 28.8(\mathrm{t}) ; 43.0(\mathrm{t}) ; 51.0(\mathrm{q}) ; 55.3(\mathrm{q}) ; 55.6(\mathrm{q}) ; 60.9$ (q); 102.9 (d); 108.4 (d); 113.5 (d); 113.7 (d); 115.8 (s); 117.6 (d); 118.9 (d); 120.3 (s); 121.6 (s); 123.6 (d); 126.1 (d); 126.8 (s); 127.0 (s); 129.3 (s); 131.6 (s); 135.3 (s); 137.2 (s); 144.8 (s); 144.9 (s); 147.5 (s); 151.4 (s); 155.5 (s); 162.3 (s). MS (MALDI-TOF) 517 (M, 100). HRMS m/z calcd. for $\mathrm{C}_{29} \mathrm{H}_{27} \mathrm{NO}_{8}$ 517.1737, found 517.1731.

\section{Methyl 8-hydroxy-9-methoxy-1-(3,4,5-trimethoxyphenyl)pyrrolo[2,1-a]isoquinoline-3-carboxylate}

(16a). Following the general procedure $\mathrm{G}$ and starting from 10a $(23 \mathrm{mg}, 0.05 \mathrm{mmol})$, elution with hexane/ AcOEt (80:20 to 70:30) gave a yellowish solid (13 mg, 70\%). M. p. (MeCN) 212-213 ${ }^{\circ} \mathrm{C}$. IR (film) v 3409, 1678, $1207 \mathrm{~cm}^{-1} .{ }^{1} \mathrm{H} \mathrm{NMR}\left(\mathrm{CDCl}_{3}, 400 \mathrm{MHz}\right) \delta 3.57$ (s, 3H, OMe); 3.86 (s, 6H, 2OMe); 3.91 (s, 3H, OMe); 3.92 (s, 3H, OMe); 5.82 (bs, 1H, OH); 6.75 (s, 2H, H2', H6'); 6.94 (d, J = 7.6 Hz, 
1H, H6); $7.14(\mathrm{~s}, 1 \mathrm{H}) ; 7.31(\mathrm{~s}, 1 \mathrm{H}) ; 7.42(\mathrm{~s}, 1 \mathrm{H}) ; 9.22(\mathrm{~d}, J=7.6 \mathrm{~Hz}, 1 \mathrm{H}, \mathrm{H} 5) .{ }^{13} \mathrm{C} \mathrm{NMR}\left(\mathrm{CDCl}_{3}, 100\right.$ MHz): $\delta 51.2(\mathrm{q}) ; 55.5$ (q); 56.2 (2q); 60.9 (q); 104.5 (d); 107.4 (2d); 110.5 (d); 112.5 (d); 114.1 (s); 118.1 (s); 119.4 (s); 121.8 (d); 123.5 (s); 124.1 (d); 130.8 (s); 132.9 (s); 137.2 (s); 146.0 (s); 146.7 (s); 153.3 (2s); 161.8 (s). MS (EI) m/z 393 (M, 100); 394 (M+1, 12). MS (ESI-TOF) $m / z 438$ (M+1, 100). HRMS $m / z$ calcd. for $\mathrm{C}_{24} \mathrm{H}_{24} \mathrm{NO}_{7} 438.1547$, found 438.1547 .

\section{Methyl 8-hydroxy-1-(4-hydroxy-3-methoxyphenyl)-9-methoxypyrrolo[2,1-a]isoquinoline-3-}

carboxylate (16c). Following the general procedure $\mathrm{G}$ and starting from 10d (23 $\mathrm{mg}, 0.05 \mathrm{mmol})$, elution with hexane/ AcOEt (80:20 to 70:30) gave a yellowish solid (13 mg, 70\%). M. p. (MeCN) 163-

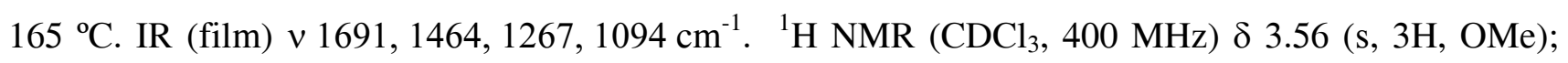
3.90 (s, 3H, OMe); 3.91 (s, 3H, OMe); 5.71 (bs, 1H, OH); 5.84 (bs, 1H, OH); 6.92 (d, J = 7.6 Hz, 1H, H6); 7.00 (d, J = 1.2 Hz, 1H, H2'); 7.03-7.05 (m, 2H, H5', H6'); 7.12 (s, 1H); 7.33 (s, 1H); 7.39 (s, 1H); $9.21(\mathrm{~d}, J=7.6 \mathrm{~Hz}, 1 \mathrm{H}, \mathrm{H} 5) .{ }^{13} \mathrm{C} \mathrm{NMR}\left(\mathrm{CDCl}_{3}, 100 \mathrm{MHz}\right): \delta 51.1(\mathrm{q}) ; 55.5$ (q); 56.0 (q); 104.5 (d); 110.5 (d); 112.3 (d); 113.0 (d); 114.0 (s); 114.3 (d); 116.2 (d); 118.1 (s); 119.6 (s); 122.2 (d); 123.5 (d); 124.0 (s); 129.1 (s); 130.9 (s); 144.9 (s); 145.9 (s); 146.5 (s); 146.7 (s); 161.8 (s). MS (EI) m/z 393 (M, 100); 394 (M+1, 12). HRMS m/z calcd. for $\mathrm{C}_{27} \mathrm{H}_{19} \mathrm{~N}_{3} \mathrm{O}_{8}$ 393.1212, found 393.1215.

\section{Methyl 1-(2,5-dimethoxyphenyl)-8-hydroxy-9-methoxypyrrolo[2,1-a]isoquinoline-3-carboxylate}

(16e). Following the general procedure $\mathrm{G}$ and starting from 10e $(26 \mathrm{mg}, 0.06 \mathrm{mmol})$, elution with hexane/ AcOEt (80:20 to 70:30) gave a yellow solid (14 mg, 57\%). M. p. (MeCN) 198-199 ${ }^{\circ} \mathrm{C}$. IR (film) v 1690, 1465, $1206 \mathrm{~cm}^{-1} .{ }^{1} \mathrm{H} \mathrm{NMR}\left(\mathrm{CDCl}_{3}, 400 \mathrm{MHz}\right) \delta 3.53$ (s, 3H, OMe); 3.64 (s, 3H, OMe); 3.79 (s, 3H, OMe); 3.90 (s, 3H, OMe); 5.80 (s, 1H, OH); 6.93 (d, $J=7.6 \mathrm{~Hz}, 1 \mathrm{H}, \mathrm{H} 6) ; 6.94$ (dd, $J=8.4$, $\left.2.8 \mathrm{~Hz}, 1 \mathrm{H}, \mathrm{H} 4^{\prime}\right) ; 6.97$ (d, J = 8.4 Hz, 1H, H3'); 6.99 (d, J=2.8 Hz, 1H, H6'); 7.12 (s, 1H); 7.14 (s, $1 \mathrm{H}) ; 7.43(\mathrm{~s}, 1 \mathrm{H}) ; 9.23(\mathrm{~d}, J=7.6 \mathrm{~Hz}, 1 \mathrm{H}, \mathrm{H} 5) .{ }^{13} \mathrm{C} \mathrm{NMR}\left(\mathrm{CDCl}_{3}, 100 \mathrm{MHz}\right): \delta 51.1(\mathrm{q}) ; 55.4(\mathrm{q}) ; 55.8$ (q); 56.3 (q); 104.4 (d); 110.2 (d); 112.1 (d); 112.4 (d); 113.4 (s); 114.0 (d); 114.3 (s); 117.9 (d); 120.0 (s); $122.4(\mathrm{~d}) ; 123.6$ (d); 124.0 (s); 127.0 (s); 131.5 (s); 145.8 (s); 146.8 (s); 152.2 (s); 153.6 (s); 161.8 
(s). MS (MALDI-TOF) $m / z 407$ (M, 100); $408(\mathrm{M}+1,40)$. HRMS $m / z$ calcd. for $\mathrm{C}_{23} \mathrm{H}_{21} \mathrm{NO}_{6} 407.1369$, found 407.1363 .

\section{Methyl 8-hydroxy-9-methoxy-1-(2-thienyl)-5,6-dihydropyrrolo[2,1-a]isoquinoline-3-carboxylate}

(16n). Following the general procedure $\mathrm{G}$ and starting from 6n (15 mg, $0.04 \mathrm{mmol})$, elution with hexane/ AcOEt (90:10) gave a white solid (6 mg, 45\%). M. p. (MeCN) 134-136 ${ }^{\circ} \mathrm{C}$. IR (film) v 3420, 1693, 1466, $1207 \mathrm{~cm}^{-1} .{ }^{1} \mathrm{H}$ NMR $\left(\mathrm{CDCl}_{3}, 200 \mathrm{MHz}\right) \delta 3.62(\mathrm{~s}, 3 \mathrm{H}, \mathrm{OMe}) ; 3.91(\mathrm{~s}, 3 \mathrm{H}, \mathrm{OMe}) ; 5.82(\mathrm{~s}$, 1H, OH); $6.95(\mathrm{~d}, J=7.5 \mathrm{~Hz}, 1 \mathrm{H}, \mathrm{H} 6) ; 7.14(\mathrm{~s}, 1 \mathrm{H}) ; 7.16(\mathrm{~s}, 1 \mathrm{H}) ; 7.17(\mathrm{~d}, J=2.0 \mathrm{~Hz}, 1 \mathrm{H}) ; 7.34-7.35$ (bd, 1H); $7.44(\mathrm{dd}, J=4.1,2.0 \mathrm{~Hz}, 1 \mathrm{H}) ; 7.48(\mathrm{~s}, 1 \mathrm{H}) ; 9.22$ (d, $J=7.5 \mathrm{~Hz}, 1 \mathrm{H}, \mathrm{H} 5) .{ }^{13} \mathrm{C} \mathrm{NMR}\left(\mathrm{CDCl}_{3}\right.$, $100 \mathrm{MHz}): \delta 51.2(\mathrm{q}) ; 55.5$ (q); 104.3 (d); 109.1 (s); 110.4 (d); 112.7 (d); 114.3 (s); 119.3 (s); 123.4 (d); 123.5 (d); 124.2 (s); 126.2 (d); 127.3 (d); 128.0 (d); 132.0 (s); 138.3 (s); 146.2 (s); 146.9 (s); 161.7 (s). MS (MALDI-TOF) m/z 353 (M, 100). MS (ESI-TOF) $m / z 354$ (M+1, 100). HRMS m/z calcd. for $\mathrm{C}_{19} \mathrm{H}_{16} \mathrm{NO}_{4} \mathrm{~S} 354.0795$, found 354.0795.

Methyl 8-hydroxy-9-methoxy-1,2-bis(3,4,5-trimethoxyphenyl)pyrrolo[2,1-a]isoquinoline-3carboxylate (17a). Following the general procedure $\mathrm{G}$ and starting from 11a $(41 \mathrm{mg}, 0.06 \mathrm{mmol})$, elution with hexane/ AcOEt (60:40 to 40:60) gave a reddish oil (25 mg, 65\%). IR (film) v 3404, 1682, 1377, $1235 \mathrm{~cm}^{-1} .{ }^{1} \mathrm{H} \mathrm{NMR}\left(\mathrm{CDCl}_{3}, 400 \mathrm{MHz}\right) \delta 3.51$ (s, 3H, OMe); 3.69 (s, 6H, 2OMe); $3.70(\mathrm{~s}, 6 \mathrm{H}$, 2OMe); 3.72 (s, 3H, OMe); 3.84 (s, 3H, OMe); 3.85 (s, 3H, OMe); 5.82 (bs, 1H, OH); 6.46 (s, 2H); 6.53 (s, 2H); $6.95(\mathrm{~d}, J=7.6 \mathrm{~Hz}, 1 \mathrm{H}, \mathrm{H} 6) ; 7.14(\mathrm{~s}, 1 \mathrm{H}) ; 7.15(\mathrm{~s}, 1 \mathrm{H}) ; 9.30(\mathrm{~d}, J=7.6 \mathrm{~Hz}, 1 \mathrm{H}, \mathrm{H} 5) .{ }^{13} \mathrm{C}$ NMR $\left(\mathrm{CDCl}_{3}, 100 \mathrm{MHz}\right) \delta 50.9$ (q); 55.3 (q); 56.0 (2q); 56.2 (2q); 60.88 (q); 60.92 (q); 104.8 (d); 108.3 (2d); 108.9 (2d); 110.4 (d); 111.8 (s); 112.4 (d); 118.2 (s); 119.0 (s); 123.6 (d); 124.4 (s); 126.9 (s); 130.5 (s); 131.7 (s); 135.3 (s); 136.8 (s); 137.1 (s); 146.0 (s); 146.7 (s); 152.0 (2s); 153.2 (2s); 162.4 (s). MS (MALDI-TOF) m/z $603(\mathrm{M}, 100) ; 604(\mathrm{M}+1,80)$. HRMS $\mathrm{m} / z$ calcd. for $\mathrm{C}_{33} \mathrm{H}_{33} \mathrm{NO}_{10}$ 603.2105, found 603.2099.

Methyl 8-hydroxy-1,2-bis(4-hydroxy-3-methoxyphenyl)-9-methoxypyrrolo[2,1-a]isoquinoline-3carboxylate (17c). Following the general procedure $\mathrm{G}$ and starting from 11c (46 $\mathrm{mg}, 0.08 \mathrm{mmol})$, a 
yellow solid (26 mg, 61\%). M. p. (MeCN) 235-237 ${ }^{\circ} \mathrm{C}$. IR (film) $\vee 3415,1680,1376,1211 \mathrm{~cm}^{-1} .{ }^{1} \mathrm{H}$ NMR $\left(\mathrm{CDCl}_{3}, 400 \mathrm{MHz}\right) \delta 3.49$ (s, 3H, OMe); 3.67 (s, 3H, OMe); 3.68 (s, 3H, OMe); 3.69 (s, 3H, OMe); 5.50 (bs, 1H, OH); 5.58 (bs, 1H, OH); 5.79 (bs, 1H, OH); 6.66 (d, J = $1.6 \mathrm{~Hz}, 2 \mathrm{H}, \mathrm{H} 2$ ', H2”); 6.72-6.77 (m, 2H, H6', H6”); 6.80 (d, $J=8.0 \mathrm{~Hz}, 1 \mathrm{H}, \mathrm{H6}) ; 6.91$ and 6.92 (2d, $J=8.6 \mathrm{~Hz}, 2 \mathrm{H}, \mathrm{H} 5^{\prime}$,

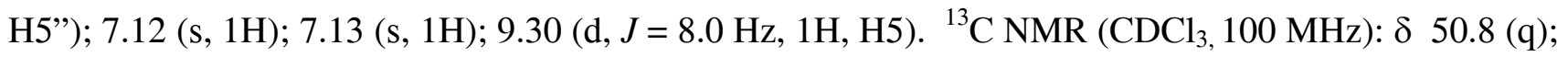
55.4 (q); 55.8 (q); 56.0 (q); 104.8 (d); 110.3 (d); 112.1 (d); 113.2 (d); 113.5 (d); 114.2 (d); 114.4 (d); 118.4 (s); 119.3 (s); 123.7 (d); 124.1 (d); 124.3 (s); 125.0 (d); 127.2 (s); 128.0 (s); 130.6 (s); 135.8 (s); 144.2 (s); 144.7 (s); 145.3 (s); 145.9 (s); 146.4 (s); 146.5 (s); 162.6 (s). MS (MALDI-TOF) 515 (M, 100); $516(\mathrm{M}+1,80)$. HRMS m/z calcd. for $\mathrm{C}_{29} \mathrm{H}_{25} \mathrm{NO}_{8}$ 515.1580, found 515.1575.

\section{Methyl}

\section{1,2-bis(3,4-dimethoxyphenyl)-8-hydroxy-9-methoxypyrrolo[2,1-a]isoquinoline-3-}

carboxylate (17f). Following the general procedure $\mathrm{G}$ and starting from 11f (22 $\mathrm{mg}, 0.04 \mathrm{mmol})$, elution with hexane/ AcOEt (60:40 to 40:60) gave a yellowish oil (10 mg, 49\%). IR (film) v 3342, $1599 \mathrm{~cm}^{-1} .{ }^{1} \mathrm{H}$ NMR $\left(\mathrm{CDCl}_{3}, 400 \mathrm{MHz}\right) \delta 3.47$ (s, 3H, OMe); 3.68 (s, 3H, OMe); 3.70 (s, 3H, OMe); 3.71 (s, 3H, OMe); 3.87 (s, 3H, OMe); 3.88 (s, 3H, OMe); 5.79 (bs, 1H, OH); 6.70-6.78 (m, 4H); $6.85(\mathrm{~s}, 2 \mathrm{H}) ; 6.92(\mathrm{~d}, J=7.6 \mathrm{~Hz}, 1 \mathrm{H}, \mathrm{H} 6) ; 7.11(\mathrm{~s}, 1 \mathrm{H}) ; 7.12(\mathrm{~s}, 1 \mathrm{H}) ; 9.29$ (d, J = $7.6 \mathrm{~Hz}, 1 \mathrm{H}, \mathrm{H} 5) .{ }^{13} \mathrm{C}$ $\operatorname{NMR}\left(\mathrm{CDCl}_{3}, 100 \mathrm{MHz}\right) \delta 50.7$ (q); $55.3(\mathrm{q}) ; 55.6(\mathrm{q}) ; 55.7$ (q); 55.8 (q); 55.9 (q); 104.8 (d); 109.9 (d); 110.3 (d); 111.1 (d); 111.9 (s); 112.1 (d); 114.2 (d); 115.0 (d), 118.3 (s); 119.3 (s), 123.2 (d); 123.7 (d); 124.2 (d); 124.3 (s); 127.8 (s); 128.7 (s); 130.6 (s); 135.7 (s); 145.9 (s); 146.5 (s); 147.5 (s); 147.5 (s); $148.0(\mathrm{~s}) ; 148.9$ (s); 162.6 (s). MS (MALDI-TOF) $\mathrm{m} / \mathrm{z} 543(\mathrm{M}) ; 544(\mathrm{M}+1)$. HRMS m/z calcd. for $\mathrm{C}_{31} \mathrm{H}_{29} \mathrm{NO}_{8}$ 543.1893, found 543.1888.

\section{Methyl 8-hydroxy-9-methoxy-1,2-bis(4-methoxyphenyl)pyrrolo[2,1-a]isoquinoline-3-carboxylate}

(17g). Following the general procedure $\mathrm{G}$ and starting from $11 \mathrm{~g}$ (42 $\mathrm{mg}, 0.08 \mathrm{mmol}$ ), elution with hexane/ AcOEt (60:40 to 40:60) gave a pale solid (27 mg, 71\%). M. p. (MeCN) 241-4 ${ }^{\circ} \mathrm{C}$. IR (film) $\vee 2951,1676 \mathrm{~cm}^{-1} .{ }^{1} \mathrm{H}$ NMR (DMSO-d $\left.6,400 \mathrm{MHz}\right) \delta 3.28$ (s, 3H, OMe); 3.54 (s, 3H, OMe); $3.71(\mathrm{~s}$, 3H, OMe); $3.74(\mathrm{~s}, 3 \mathrm{H}, \mathrm{OMe}) ; 6.77(\mathrm{~d}, J=8.6 \mathrm{~Hz}, 2 \mathrm{H}) ; 6.80(\mathrm{~s}, 1 \mathrm{H}) ; 6.96(\mathrm{~d}, J=8.6 \mathrm{~Hz}, 2 \mathrm{H}) ; 7.08-7.11$ 
(m, 3H); $7.11(\mathrm{~s}, 1 \mathrm{H}) ; 7.19(\mathrm{~d}, J=8.5 \mathrm{~Hz}, 2 \mathrm{H}) ; 9.14(\mathrm{~d}, J=7.6 \mathrm{~Hz}, 1 \mathrm{H}, \mathrm{H} 5) ; 9.67$ (bs, $1 \mathrm{H}, \mathrm{OH}) .{ }^{13} \mathrm{C}$ NMR (DMSO-d6, 100 MHz) $\delta 50.5$ (q); 54.3 (q); 54.7 (q); 55.0 (q); 104.7 (d), 111.1 (d); 111.9 (d); 112.3 (2d); 113.8 (2d); 117.6 (s); 117.8 (s), 122.5 (s); 123.4 (d); 126.9 (s); 127.4 (s); 130.0 (s); 131.3 (2d); 132.8 (2d); 134.9 (s); 147.2 (s); 148.0 (s); 157.6 (s); 158.3 (s); 161.6 (s). MS (MALDI-TOF) $\mathrm{m} / \mathrm{z}$ 483 (M). HRMS $m / z$ calcd. for $\mathrm{C}_{29} \mathrm{H}_{25} \mathrm{NO}_{6} 483.1682$, found. 483.1676.

\section{Methyl 8-hydroxy-9-methoxy-1,2-bis(4-trifluoromethoxyphenyl)pyrrolo[2,1-a]isoquinoline-3-}

carboxylate (17i). Following the general procedure $\mathrm{G}$ and starting from 11i (29 $\mathrm{mg}, 0.05 \mathrm{mmol})$, elution with hexane/ AcOEt (60:40 to 40:60) gave a reddish oil $(8 \mathrm{mg}, 30 \%)$. IR (film) $v 1727 \mathrm{~cm}^{-1} .{ }^{1} \mathrm{H}$ NMR (Acetone-d $\left.\mathrm{d}_{6}, 400 \mathrm{MHz}\right) \delta 3.26(\mathrm{~s}, 3 \mathrm{H}, \mathrm{OMe}) ; 3.46$ (s, 3H, OMe); 6.75 (s, 1H); 7.01 (d, J= $7.6 \mathrm{~Hz}$, 1H, H6); $7.06(\mathrm{~d}, J=8.0 \mathrm{~Hz}, 2 \mathrm{H}) ; 7.09(\mathrm{~s}, 1 \mathrm{H}) ; 7.23(\mathrm{~d}, J=8.7 \mathrm{~Hz}, 2 \mathrm{H}) ; 7.26(\mathrm{~d}, J=8.0 \mathrm{~Hz}, 2 \mathrm{H}) ; 7.36$ $(\mathrm{d}, J=8.7 \mathrm{~Hz}, 2 \mathrm{H}) ; 8.23(\mathrm{bs}, 1 \mathrm{H}, \mathrm{OH}) ; 9.18(\mathrm{~d}, J=7.6 \mathrm{~Hz}, 1 \mathrm{H}, \mathrm{H} 5) .{ }^{13} \mathrm{C}$ NMR (Acetone-d $\left.6,100 \mathrm{MHz}\right)$ $\delta 49.4(\mathrm{q}) ; 53.5$ (q); 103.9 (d), 110.6 (d); 111.5 (q); 111.9 (d); 116.0 (q); 117.7 (s); 118.8 (2d); 120.6 (2d); 122.2 (s); 123.6 (s); 129.7 (d); 131.6 (s); 133.1 (2d); 133.5 (2d); 133.9 (s); 134.7 (s); 146.9 (s); 147.1 (s); 147.5 (s); 147.6 (s); 161.0 (s). MS (MALDI-TOF) $\mathrm{m} / \mathrm{z} 591$ (M). HRMS $\mathrm{m} / \mathrm{z}$ calcd. for $\mathrm{C}_{29} \mathrm{H}_{19} \mathrm{~F}_{6} \mathrm{NO}_{6}$ 591.1117, found. 591.1111.

\section{Methyl 8-hydroxy-1,2-bis(4-hydroxyphenyl)-9-methoxypyrrolo[2,1-a]isoquinoline-3-carboxylate}

(17j). Following the general procedure $G$ and starting from $\mathbf{1 1 j}(77 \mathrm{mg}, 0.18 \mathrm{mmol}$ ), elution with hexane/ AcOEt (60:40 to 40:60) gave a pale solid (32 mg, 53\%). M. p. (MeCN) 280-4 ${ }^{\circ} \mathrm{C}$. IR (film) v 3373, $1684 \mathrm{~cm}^{-1} .{ }^{1} \mathrm{H}$ NMR (DMSO-d $\left.6,400 \mathrm{MHz}\right) \delta 3.29$ (s, 3H, OMe); 3.54 (s, 3H, OMe); 6.58 (d, $J$ $=8.5 \mathrm{~Hz}, 2 \mathrm{H}) ; 6.77(\mathrm{~d}, J=8.5 \mathrm{~Hz}, 2 \mathrm{H}) ; 6.90(\mathrm{~s}, 1 \mathrm{H}) ; 6.95(\mathrm{~d}, J=8.5 \mathrm{~Hz}, 2 \mathrm{H}) ; 7.03(\mathrm{~s}, 1 \mathrm{H}) ; 7.05-7.08$ $(\mathrm{m}, 3 \mathrm{H}) ; 9.12$ (d, $J=7.6 \mathrm{~Hz}, 1 \mathrm{H}, \mathrm{H} 5) ; 9.22$ (bs, 1H, OH); 9.42 (bs, 1H, OH); 9.64 (bs, $1 \mathrm{H}, \mathrm{OH}) .{ }^{13} \mathrm{C}$ NMR (DMSO-d 6 , 100 MHz) $\delta 50.4$ (q); 54.3 (q); 104.9 (d); 110.9 (d); 111.7 (d); 113.8 (2d); 115.2 (2d); 117.9 (s); 118.0 (s), 122.5 (s); 123.3 (d); 125.3 (s); 125.7 (s); 130.0 (s); 131.3 (2d); 132.7 (2d); 135.5 (s); 147.1 (s); 147.9 (s); 155.7 (s); 156.4 (s); 161.7 (s). MS (MALDI-TOF) $m / z 455$ (M, 100). HRMS $m / z$ calcd. for $\mathrm{C}_{27} \mathrm{H}_{21} \mathrm{NO}_{6} 455.1369$, found 455.1363 . 


\section{Methyl 8-hydroxy-1,2-bis(3-hydroxyphenyl)-9-methoxypyrrolo[2,1-a]isoquinoline-3-carboxylate}

$\left(\mathbf{1 7 k}, \mathbf{R}^{\mathbf{3}}=\mathbf{O H}\right)$. Following the general procedure $\mathrm{G}$ and starting from $\mathbf{1 1 k}(67 \mathrm{mg}, 0.12 \mathrm{mmol})$, elution with hexane/ AcOEt (60:40 to 40:60) gave a pale solid (24 mg, 46\%). M. p. (MeCN) $260-265^{\circ} \mathrm{C}$. IR (film) v 3384, $1653 \mathrm{~cm}^{-1} .{ }^{1} \mathrm{H}$ NMR (DMSO-d $\left.6,400 \mathrm{MHz}\right) \delta 3.29(\mathrm{~s}, 3 \mathrm{H}, \mathrm{OMe}) ; 3.53\left(\mathrm{~s}, 3 \mathrm{H}, \mathrm{CO}_{2} \mathrm{Me}\right)$; 6.66-6.76 (m, 3H); $6.72(\mathrm{t}, J=1.8 \mathrm{~Hz}, 1 \mathrm{H}) ; 6.70-6.72(\mathrm{dd}, J=7.8,1.9 \mathrm{~Hz}, 2 \mathrm{H}) ; 6.90(\mathrm{~s}, 1 \mathrm{H}) ; 6.98-7.02$ $(\mathrm{dd}, J=8.7,9.0 \mathrm{~Hz}, 1 \mathrm{H}) ; 7.10(\mathrm{~s}, 1 \mathrm{H}) ; 7.11(\mathrm{~d}, J=7.6 \mathrm{~Hz}, 1 \mathrm{H}, \mathrm{H6}) ; 7.19(\mathrm{t}, J=8.0 \mathrm{~Hz}, 1 \mathrm{H}) ; 9.11(\mathrm{~d}, J$ $=7.6 \mathrm{~Hz}, 1 \mathrm{H}, \mathrm{H} 5) ; 9.17$ (bs, $1 \mathrm{H}, \mathrm{OH}) ; 9.27$ (bs, 1H, OH); 9.40 (bs, 1H, OH). ${ }^{13} \mathrm{C}$ NMR (DMS-d, 100 MHz) $\delta 50.6$ (q); 54.3 (q); 104.9 (d), 111.1 (d); 112.1 (d); 113.4 (d); 114.1 (d); 117.2 (d); 117.7 (s); 117.8 (s); 118.2 (d); 121.1 (d); 122.3 (d); 122.4 (d); 123.4 (s); 127.7 (d); 129.3 (d); 129.5 (s); 134.9 (s); 136.1 (s); 136.7 (s); 147.2 (s); 148.1 (s); 155.8 (s); 157.2 (s); 161.6 (s). MS (MALDI-TOF) m/z 455 (M). HRMS $m / z$ calcd. for $\mathrm{C}_{27} \mathrm{H}_{21} \mathrm{NO}_{6} 455.1369$, found. 455.1363 .

\section{Methyl 8-hydroxy-9-methoxy-1,2-bis(3-nitrophenyl)pyrrolo[2,1-a]isoquinoline-3-carboxylate}

(17m). Following the general procedure $\mathrm{G}$ and starting from $\mathbf{1 1 m}(31 \mathrm{mg}, 0.06 \mathrm{mmol})$, elution with hexane/ AcOEt (60:40 to 40:60) gave a pale solid $(23 \mathrm{mg}, 82 \%)$. M. p. $(\mathrm{MeOH})=185-190{ }^{\circ} \mathrm{C}$. IR (film) v 1689, 1537, 1379, $1348 \mathrm{~cm}^{-1} .{ }^{1} \mathrm{H}$ NMR (DMSO-d 6 , $\left.400 \mathrm{MHz}\right) 3.25$ (s, 3H, OMe); 3.56 (s, 3H, $\left.\mathrm{CO}_{2} \mathrm{Me}\right) ; 6.76(\mathrm{~s}, 1 \mathrm{H}) ; 7.19(\mathrm{~s}, 1 \mathrm{H}) ; 7.25(\mathrm{~d}, J=7.6 \mathrm{~Hz}, 1 \mathrm{H}, \mathrm{H} 6) ; 7.53(\mathrm{td}, J=7.6,1.2 \mathrm{~Hz}, 1 \mathrm{H})$; 7.67$7.72(\mathrm{~m}, 2 \mathrm{H}) ; 7.85(\mathrm{dt}, J=8.0,1.2 \mathrm{~Hz}, 1 \mathrm{H}) ; 8.08(\mathrm{~d}, J=1.2 \mathrm{~Hz}, 1 \mathrm{H}) ; 8.07(\mathrm{dt}, J=8.0,1.2 \mathrm{~Hz}, 1 \mathrm{H})$; 8.16-8.19 (m, 2H); 9.22 (d, $J=7.6 \mathrm{~Hz}, 1 \mathrm{H}, \mathrm{H} 5) .{ }^{13} \mathrm{C}$ NMR (DMSO-d $\left.6,100 \mathrm{MHz}\right) \delta 50.9$ (q); 54.4 (q); 104.3 (d), 111.5 (d); 111.8 (s); 113.1 (d); 115.2 (s); 117.2 (s), 121.7 (d); 122.3 (d); 122.4 (d); 123.7 (s); 125.0 (d); 126.0 (d); 128.7 (d); 129.9 (d); 130.0 (s); 132.7 (s); 136.2 (s); 136.6 (s); 137.2 (d); 138.5 (d); 146.8 (s); 147.7 (s); 147.9 (s); 148.5 (s); 160.9 (s). MS (MALDI-TOF) m/z 513 (M); 514 (M+1). HRMS m/z calcd. for $\mathrm{C}_{27} \mathrm{H}_{19} \mathrm{~N}_{3} \mathrm{O}_{8}$ 513.1172, found 513.1167.

\section{Methyl 8-hydroxy-9-methoxy-1,2-bis(2-thienyl)pyrrolo[2,1-a]isoquinoline-3-carboxylate (17n).}

Following the general procedure $\mathrm{G}$ and starting from $11 \mathrm{n}$ (12 $\mathrm{mg}, 0.03 \mathrm{mmol})$, elution with hexane/ AcOEt (90:10 to 75:25) gave a pale solid (5 mg, 40\%). M. p. (MeCN) 205-208 ${ }^{\circ} \mathrm{C} . \quad$ IR (film) $\vee 3409$, 
1683, 1434, 1376, $1246 \mathrm{~cm}^{-1} .{ }^{1} \mathrm{H}$ NMR $\left(\mathrm{CDCl}_{3}, 400 \mathrm{MHz}\right) \delta 3.56$ (s, 3H, OMe); 3.73 (s, 3H, OMe); $5.82(\mathrm{bs}, 1 \mathrm{H}, \mathrm{OH}) ; 6.93-6.95(\mathrm{~m}, 2 \mathrm{H}) ; 6.96$ (d, $J=7.6 \mathrm{~Hz}, 1 \mathrm{H}, \mathrm{H6}) ; 7.05$ (dd, $J=3.4,1.2 \mathrm{~Hz}, 1 \mathrm{H}) ; 7.08-$ $7.11(\mathrm{~m}, 2 \mathrm{H}) ; 7.13(\mathrm{~s}, 1 \mathrm{H}) ; 7.26-7.28(\mathrm{~m}, 1 \mathrm{H}) ; 7.39(\mathrm{dd}, J=5.2,1.2,1 \mathrm{H}) ; 9.26(\mathrm{~d}, J=7.6 \mathrm{~Hz}, 1 \mathrm{H}, \mathrm{H} 5)$. ${ }^{13} \mathrm{C} \mathrm{NMR}\left(\mathrm{CDCl}_{3}, 100 \mathrm{MHz}\right): \delta 51.0(\mathrm{q}) ; 55.3(\mathrm{q}) ; 104.6(\mathrm{~d}) ; 110.3(\mathrm{~d}) ; 110.4(\mathrm{~s}) ; 112.9(\mathrm{~d}) ; 113.2(\mathrm{~s}) ;$ 118.9 (s); 123.4 (d); 124.4 (s); 125.8 (d); 126.0 (d); 127.19 (d); 127.24 (d); 128.3 (d); 129.5 (s); 129.9 (d); 131.8 (s); 135.0 (s); 136.7 (s); 146.2 (s); 146.9 (s); 162.2 (s). MS (ESI) $m / z, 436$ (M+1, 100); 437 $(\mathrm{M}+2,65)$. MS (ESI-TOF) $m / z 436(\mathrm{M}+1,100)$. HRMS $m / z$ calcd. for $\mathrm{C}_{23} \mathrm{H}_{18} \mathrm{NO}_{4} \mathrm{~S}_{2} 436.0672$, found 436.0672 .

\section{Methyl 8-hydroxy-2-(4-hydroxyphenyl)-1-(4-hydroxy-3-methoxyphenyl)-9-methoxypyrrolo[2,1-}

a]isoquinoline-3-carboxylate (18a). Following the general procedure $\mathrm{G}$ and starting from $\mathbf{1 2 a}$ (82 $\mathrm{mg}$, $0.14 \mathrm{mmol}$ ), elution with hexane/ AcOEt (60:40 to 40:60) gave a pinkish solid (62 mg, 89\%). M. p. $(\mathrm{MeCN}) 260-262{ }^{\circ} \mathrm{C}$. IR (film) $v 3364,1653 \mathrm{~cm}^{-1} .{ }^{1} \mathrm{H}$ NMR $\left(\mathrm{MeOD}-\mathrm{d}_{4}, 400 \mathrm{MHz}\right) \delta 3.43(\mathrm{~s}, 3 \mathrm{H}$, OMe); 3.60 (s, 3H, OMe); 3.67 (s, 3H, OMe); 6.63 (d, J = 8.8 Hz, 2H, H3”, H5”); 6.69-6.72 (m, 2H, H2', H6'); 6.81 (bd, $J=8.0$ Hz, 1H, H5'); 6.88 (d, $J=7.6 \mathrm{~Hz}, 1 \mathrm{H}, \mathrm{H6}$ ); 6.98 (d, $J=8.8 \mathrm{~Hz}, 2 \mathrm{H}, \mathrm{H} 2$ ', H6”); 7.02 (s, 1H); 7.10 (s, 1H); 9.18 (d, J=7.6 Hz, 1H, H5). ${ }^{13} \mathrm{C}$ NMR (MeOD-d, $\left.100 \mathrm{MHz}\right) \delta 51.0$ (q); 55.7 (q); 56.5 (q); 106.5 (d); 112.1 (d); 112.8 (s); 112.9 (d); 114.9 (2d); 116.3 (d); 116.8 (d); 120.1 (s); 120.2 (s); 124.3 (d); 125.6 (s); 126.0 (d); 128.2 (s); 128.9 (s); 132.2 (s); 132.9 (2d); 137.8 (s); 146.8 (s); 148.4 (s); 149.0 (s); 149.3 (s); 157.1 (s); 164.2 (s). MS (ESI-TOF) 486 (M+1, 67); 486 (MNa+, 100). HRMS $m / z$ calcd. for $\mathrm{C}_{28} \mathrm{H}_{23} \mathrm{NNaO}_{7}^{+}$508.1367, found 508.1367.

Methyl

2-(3,4-dimethoxyphenyl)-8-hydroxy-1-(4-hydroxy-3-methoxyphenyl)-9-

methoxypyrrolo[2,1-a]isoquinoline-3-carboxylate (18b). Following the general procedure $G$ and starting from 12b (66 mg, $0.11 \mathrm{mmol}$ ), elution with hexane/ AcOEt (60:40 to 40:60) gave a yellowish solid (38 mg, 67\%). M. p. (MeCN) $110-113{ }^{\circ} \mathrm{C}$. IR (film) v 3420, $1676 \mathrm{~cm}^{-1} .{ }^{1} \mathrm{H}$ NMR $\left(\mathrm{CDCl}_{3}, 400\right.$ MHz) $\delta 3.49(\mathrm{~s}, 3 \mathrm{H}, \mathrm{OMe}) ; 3.68(\mathrm{~s}, 3 \mathrm{H}, \mathrm{OMe}) ; 3.69$ (s, 3H, OMe); 3.70 (s, 3H, OMe); 3.87 (s, 3H, OMe); 5.58 (bs, 1H, OH); 5.79 (bs, 1H, OH); $6.67(\mathrm{~d}, J=2.0 \mathrm{~Hz}, 1 \mathrm{H}) ; 6.72(\mathrm{~d}, J=1.6 \mathrm{~Hz}, 1 \mathrm{H}) ; 6.75(\mathrm{~d}$, 
$J=8.4 \mathrm{~Hz}, 1 \mathrm{H}) ; 6.78(\mathrm{dd}, J=8.4,2.0,1 \mathrm{H}) ; 6.89(\mathrm{dd}, J=8.0,1.6 \mathrm{~Hz}, 1 \mathrm{H}) ; 6.92(\mathrm{~d}, J=7.6 \mathrm{~Hz}, 1 \mathrm{H}, \mathrm{H} 6)$; $6.93(\mathrm{~d}, J=8.0 \mathrm{~Hz}, 1 \mathrm{H}) ; 7.117(\mathrm{~s}, 1 \mathrm{H}) ; 7.122(\mathrm{~s}, 1 \mathrm{H}) ; 9.29(\mathrm{~d}, J=7.6 \mathrm{~Hz}, 1 \mathrm{H}, \mathrm{H} 5) .{ }^{13} \mathrm{C} \mathrm{NMR}\left(\mathrm{CDCl}_{3}\right.$, $100 \mathrm{MHz}) \delta 50.8(\mathrm{q}) ; 55.4(\mathrm{q}) ; 55.7(2 \mathrm{q}) ; 56.0(\mathrm{q}) ; 104.8(\mathrm{~d}) ; 109.9(\mathrm{~d}) ; 110.3(\mathrm{~d}) ; 111.9(\mathrm{~s}) ; 112.1(\mathrm{~d}) ;$ 114.2 (2d); 114.3 (d); 118.4 (s); 119.3 (s), 123.2 (d); 123.7 (d); 124.3 (s); 125.0 (d); 127.8 (s); 128.0 (s); 130.6 (s); 135.7 (s); 144.7 (s); 145.9 (s); 147.53 (s); 146.4 (s); 146.5 (s); 147.5 (s); 162.8 (s). MS (MALDI-TOF) 529 (M, 100). HRMS m/z calcd. for $\mathrm{C}_{30} \mathrm{H}_{27} \mathrm{NO}_{8}$ 529.1737, found 529.1731.

\section{Methyl}

1-(3,5-dimethoxy-4-hydroxyphenyl)-8-hydroxy-9-methoxy-2-(3,4,5trimethoxyphenyl)pyrrolo[2,1-a]isoquinoline-3-carboxylate $\left(18 \mathrm{c} \mathrm{R}^{4}\right.$ of $\left.2-\mathrm{Ar}=\mathrm{OH}\right)$. Following the general procedure $\mathrm{G}$ and starting from $12 \mathrm{c}(80 \mathrm{mg}, 0.12 \mathrm{mmol})$ using an excess of $\mathrm{AlCl}_{3}(0.32 \mathrm{mmol})$, elution with hexane/ AcOEt (60:40 to 40:60) gave a yellowish solid (61 mg, 96\%). M. p. (MeCN) 163$166{ }^{\circ} \mathrm{C}$. IR (film) v 3421, $1678 \mathrm{~cm}^{-1} .{ }^{1} \mathrm{H}$ NMR $\left(\mathrm{CDCl}_{3}, 400 \mathrm{MHz}\right) \delta 3.49$ (s, 3H, OMe); 3.69 (s, 3H, $\mathrm{OMe}) ; 3.70$ (s, 3H, OMe); 3.72 (s, 6H, 2OMe); 5.44 (s, 1H, OH); 5.60 (s, 1H, OH); 5.80 (s, 1H, OH); 6.45 (s, 2H, H2”, H6”); 6.66 (d, $J=1.6 \mathrm{~Hz}, 1 \mathrm{H}, \mathrm{H} 2$ '); 6.91 (dd, $J=8.4,1.6 \mathrm{~Hz}, 1 \mathrm{H}, \mathrm{H6}$ '); 6.93 (d, $J=$ $7.6 \mathrm{~Hz}, 1 \mathrm{H}, \mathrm{H6}$ ); 6.95 (d, $J=8.4 \mathrm{~Hz}, 1 \mathrm{H}, \mathrm{H} 5$ '); 7.13 (s, 2H, H7, H10); 9.30 (d, $J=7.6 \mathrm{~Hz}, 1 \mathrm{H}, \mathrm{H} 5) .{ }^{13} \mathrm{C}$ $\operatorname{NMR}\left(\mathrm{CDCl}_{3}, 100 \mathrm{MHz}\right) \delta .50 .8$ (q); 55.4 (q); 56.0 (q); 56.2 (2q); 104.8 (d); 108.0 (2d); 110.3 (d); 111.7 (s); 112.2 (d); 114.20 (d); 114.23 (d), 118.3 (s); 119.2 (s), 123.6 (d); 124.3 (s); 125.0 (d); 126.2 (s); 128.1 (s); 130.6 (s); 133.4 (s); 135.7 (s); 144.7 (s); 145.90 (s); 145.93 (2s); 146.5 (s); 146.6 (s); 162.6 (s). MS (ESI-TOF) $514(\mathrm{M}, 26) ; 568(\mathrm{M}+\mathrm{Na}, 100)$. HRMS $m / z$ calcd. for $\mathrm{C}_{30} \mathrm{H}_{27} \mathrm{NNaO}_{9}{ }^{+} 568.1578$, found 568.1578 .

\section{Methyl 2-(2,4-dihydroxy-5-methoxyphenyl)-8-hydroxy-1-(4-hydropoxy-3-methoxyphenyl)-9-} methoxypyrrolo[2,1-a]isoquinoline-3-carboxylate (18d). Following the general procedure $G$ and starting from 12d (97 mg, $0.14 \mathrm{mmol}$ ), elution with hexane/ AcOEt (60:40 to 40:60) gave a light brown solid (63 mg, 86\%). M. p. (MeCN) $163-166{ }^{\circ} \mathrm{C}$. IR (film) v 3426, $1679 \mathrm{~cm}^{-1} .{ }^{1} \mathrm{H} \mathrm{NMR}\left(\mathrm{CDCl}_{3}, 400\right.$ MHz) $\delta 3.51$ (s, 3H, OMe); 3.55 (s, 3H, OMe); 3.66 (s, 3H, OMe); 3.77 (s, 3H, OMe); 5.43 (br, 1H, $\mathrm{OH}) ; 5.56(\mathrm{~s}, 1 \mathrm{H}, \mathrm{OH}) ; 5.61(\mathrm{~s}, 1 \mathrm{H}, \mathrm{OH}) ; 5.87(\mathrm{~s}, \mathrm{OH}) ; 6.33-6.92(\mathrm{~m}, 3 \mathrm{H}) ; 6.92-7.27(\mathrm{~m}, 5 \mathrm{H}) ; 9.19(\mathrm{~m}$, 
1H, H5). ${ }^{13} \mathrm{C} \mathrm{NMR}\left(\mathrm{CDCl}_{3}, 100 \mathrm{MHz}\right) \delta 51.4(\mathrm{q}) ; 55.4$ (q); 56.0 (q); 56.4 (q); 102.7 (d); 104.8 (d); 110.4 (d); 112.5 (d); 112.7 (s); 113.8 (d); 113.9 (s); 114.0 (d); 114.1 (d); 119.1 (s); 123.4 (d); 124.2 (d); 124.5 (s); 131.3 (s); 140.2 (s); 144.8 (s); 145.8 (s); 146.1 (s); 146.6 (s); 146.7 (s); 148.6 (s); 162.3 (s). MS (MALDI-TOF) $531(\mathrm{M}, 100), 532(\mathrm{M}+1,38), 533(\mathrm{M}+2,11)$. HRMS m/z calcd. for $\mathrm{C}_{29} \mathrm{H}_{25} \mathrm{NO}_{9}$ 531.1529, found 531.1524.

\section{Methyl 2-(2,5-dimethoxy-4-hydroxyphenyl)-8-hydroxy-1-(4-hydroxy-3-methoxyphenyl)-9-} methoxypyrrolo[2,1-a]isoquinoline-3-carboxylate (18e). Following the general procedure $G$ and starting from 12e $(51 \mathrm{mg}, 0.08 \mathrm{mmol})$, elution with hexane/ AcOEt (60:40 to 40:60) gave a reddish solid (21 mg, 50\%). M. p. (MeCN) $149-151^{\circ} \mathrm{C}$. IR (film) $v 3389,1681,1438,1206 \mathrm{~cm}^{-1} .{ }^{1} \mathrm{H}$ NMR $\left(\mathrm{CDCl}_{3}, 400 \mathrm{MHz}\right) \delta 3.50$ (s, 3H, OMe); 3.61 (bs, 3H, OMe); 3.65 (2s, 6H, 2OMe); 3.70 (s, 3H, OMe); 5.56 (bs, 2H, 2OH); $5.78(\mathrm{~s}, 1 \mathrm{H}, \mathrm{OH}) ; 6.51(\mathrm{~s}, 1 \mathrm{H}) ; 6.55(\mathrm{bs}, 1 \mathrm{H}) ; 6.72(\mathrm{bs}, 1 \mathrm{H}) ; 6.85-6.93(\mathrm{~m}, 3 \mathrm{H})$; $7.11(\mathrm{~s}, 1 \mathrm{H}) ; 7.18(\mathrm{~s}, 1 \mathrm{H}) ; 9.25(\mathrm{~d}, J=7.6 \mathrm{~Hz}, 1 \mathrm{H}, \mathrm{H} 5) .{ }^{13} \mathrm{C} \mathrm{NMR}\left(\mathrm{CDCl}_{3}, 100 \mathrm{MHz}\right) \delta 50.8(\mathrm{q}) ; 55.4$ (q); 55.9 (q); 56.0 (q); 56.6 (q); 98.8 (d); 104.8 (d); 110.3 (d); 111.9 (d); 114.1 (d); 115.0 (d); 115.6 (d); 118.5 (s); 119.4 (s); 123.7 (d); 124.1 (s); 124.6 (d); 124.7 (s); 128.2 (s); 130.5 (s); 139.6 (s); 144.5 (s); 145.2 (s); 145.7 (2s); 146.5 (2s); 152.0 (s); 162.6 (s). MS (MALDI-TOF) 545 (M, 100); 546 (M+1, 70). HRMS $m / z$ calcd. for $\mathrm{C}_{30} \mathrm{H}_{27} \mathrm{NO}_{9} 545.1686$, found 545.1680.

\section{Methyl 1-(2,5-dimethoxyphenyl)-8-hydroxy-9-methoxy-2-(2,4,5-trihydroxyphenyl)pyrrolo[2,1-} a]isoquinoline-3-carboxylate (18g). Following the general procedure $\mathrm{G}$ and starting from $\mathbf{1 2 g}$ (31 $\mathrm{mg}$, $0.04 \mathrm{mmol}$ ), elution with hexane/ AcOEt (60:40 to 40:60) gave a reddish solid (15 mg, 62\%). M. p. $(\mathrm{MeCN}) 149-150^{\circ} \mathrm{C}$. IR (film) v 3418, 1690, 1466, $1208 \mathrm{~cm}^{-1} .{ }^{1} \mathrm{H}$ NMR $\left(\mathrm{CDCl}_{3}, 400 \mathrm{MHz}\right) \delta 3.53(\mathrm{~s}$, 3H, OMe); 3.65 (s, 3H, OMe); 3.79 (s, 3H, OMe); 3.90 (s, OMe); 6.91-6.99 (m, 5H, H6, H7, H3', H4', H6'); 7.12 (s, 1H, H3”); 7.14 (s, 1H); 7.43 (s, 1H, H6”); 9.22 (d, J = 7.6 Hz, 1H, H5). ${ }^{13}$ C NMR $\left(\mathrm{CDCl}_{3}, 100 \mathrm{MHz}\right) \delta 51.1(\mathrm{q}) ; 53.4(\mathrm{q}) ; 55.8$ (q); 56.3 (q); 104.4 (d); 107.2 (d); 110.2 (d); 112.1 (d); 112.4 (d); 113.4 (s); 114.0 (d); 114.3 (s); 117.9 (d); 120.0 (s); 122.4 (d); 123.6 (d); 123.9 (s); 127.0 (s); 
130.8 (s); 131.5 (s); 145.8 (s); 146.8 (s); 152.2 (s); 152.8 (s); 153.4 (s); 153.6 (s); 153.7 (s); 161.8 (s). MS (MALDI-TOF) 531 (M, 100). HRMS m/z calcd. for $\mathrm{C}_{30} \mathrm{H}_{27} \mathrm{NO}_{9} 531.1529$, found 531.1527.

Methyl 2-(2,4-dihydroxyphenyl)-8-hydroxy-1-(3-hydroxyphenyl)-9-methoxypyrrolo[2,1a]isoquinoline-3-carboxylate (18h). Following the general procedure $\mathrm{G}$ and starting from $\mathbf{1 2 h}$ (27 $\mathrm{mg}$, $0.04 \mathrm{mmol}$ ), elution with hexane/ AcOEt (60:40 to 40:60) gave a yellow solid (8 mg, 40\%). M. p. 167$169{ }^{\circ} \mathrm{C} . \mathrm{IR}(\mathrm{film}) \vee 3374,1683,1207 \mathrm{~cm}^{-1} .{ }^{1} \mathrm{H} \mathrm{NMR}\left(\mathrm{CDCl}_{3}, 400 \mathrm{MHz}\right) \delta 3.57(\mathrm{~s}, 3 \mathrm{H}, \mathrm{OMe}) ; 3.91(\mathrm{~s}$, 3H, OMe); 6.86-6.89 (m, 1H); $6.91(\mathrm{~d}, J=7.6 \mathrm{~Hz}, 1 \mathrm{H}, \mathrm{H6}) ; 6.97-7.00(\mathrm{~m}, 3 \mathrm{H}) ; 6.98-7.02(\mathrm{~m}, 1 \mathrm{H}) ; 7.12$ (s, 1H); $7.34(\mathrm{t}, J=8.0 \mathrm{~Hz}, 1 \mathrm{H}, \mathrm{H} 5$ ') 7.35 (bs, $1 \mathrm{H}) ; 7.40(\mathrm{~s}, 1 \mathrm{H}) ; 9.20(\mathrm{~d}, J=7.6 \mathrm{~Hz}, 1 \mathrm{H}, \mathrm{H} 5) .{ }^{13} \mathrm{C}$ NMR $\left(\mathrm{CDCl}_{3}, 100 \mathrm{MHz}\right) \delta 51.2$ (q); 55.5 (q); 104.6 (d); 110.4 (d); 112.4 (d); 114.1 (d); 117.3 (d); 117.8 (s); 118.4 (s); 119.4 (s); 122.1 (d); 123.0 (d); 123.5 (d); 124.0 (d); 129.6 (d); 130.1 (s); 130.8 (s); 133.4 (d); 138.9 (s); 145.9 (s); 146.7 (s); 155.2 (s); 155.7 (s); 156.0 (s); 161.8 (s). MS (MALDI-TOF) 471 (M, 100). HRMS $m / z$ calcd. for $\mathrm{C}_{27} \mathrm{H}_{21} \mathrm{NO}_{7} 471.1318$, found 471.1317 .

\section{Cell growth inhibition assay: screening}

A colorimetric assay using sulforhodamine B (SRB) was adapted to perform quantitative measurement of cell growth and viability, following a previously described method. ${ }^{45}$ Cells were seeded in 96 -well microtiter plates, at $5 \times 10^{3}$ cells per well, in aliquots of $195 \mu \mathrm{L}$ of RPMI medium, and were allowed to attach to the plate surface by growing in drug-free medium for 18 hours. Afterwards, samples were added in aliquots of $5 \mu \mathrm{L}$ (dissolved in DMSO: $\mathrm{H}_{2} \mathrm{O}, 3: 7$ ). After 72 hours of exposure, the antitumor effect was measured by the SRB methodology: cells were fixed by adding $50 \mu \mathrm{L}$ of cold $50 \%$ (wt/vol) trichloroacetic acid (TCA) and were incubated for 60 minutes at $4{ }^{\circ} \mathrm{C}$. Plates were washed with deionized $\mathrm{H}_{2} \mathrm{O}$ and dried; $100 \mu \mathrm{L}$ of SRB solution (0.4\% wt/vol in $1 \%$ acetic acid) was added to each microtiter well and incubated for 10 minutes at room temperature. Unbound SRB was removed by washing with $1 \%$ acetic acid. Plates were air-dried and bound stain was solubilized with Tris buffer. Optical densities were read on an automated spectrophotometer plate reader at a single wavelength of $490 \mathrm{~nm}$. Data analyses were generated automatically by LIMS implementation. Using control OD 
values $(C)$, test $\mathrm{OD}$ values $(\mathrm{T})$ and time zero OD values $\left(\mathrm{T}_{0}\right)$, the drug concentration that causes $50 \%$ Growth Inhibition $\left(\mathrm{GI}_{50}\right.$ value) was calculated from the equation: $\left.100 \times\left[\left(\mathrm{T}-\mathrm{T}_{0}\right) / \mathrm{C}-\mathrm{T}_{0}\right)_{-}\right]=50$.

ACKNOWLEDGMENT This work was partially supported by CICYT (BQU 2003-00089), Generalitat de Catalunya, and the Barcelona Science Park. PharmaMar S. L. is also gratefully acknowledged for performing the preliminary biological tests. A. M. thanks the Junta de Andalucía, UJA and UB for financial support and for facilitating his stay.

SUPPORTING INFORMATION Experimental procedures and characterization by ${ }^{1} \mathrm{H}-{ }^{13} \mathrm{C}-\mathrm{NMR}$, HRMS and HPLC analyses of synthesized compounds, as well as, ${ }^{1} \mathrm{H}-\mathrm{NMR}$ at variable temperature and gHSQC correlations of $\mathbf{1 2 f}$ is available free of charge via the Internet at http://pubs.acs.org.

\section{REFERENCES AND NOTES}

1 Dobson, C. M. Chemical Space and Biology Nature, 2004, 432, 824-828.

2 Breinbauer, R.; Vetter, I. R.; Waldmann, H. From Protein Domains to Drug Candidates-Natural Products as Guiding Principles in the Design and Synthesis of Compound Libraries Angew. Chem. Int. Ed. 2002, 41, 2878-2890.

3 Balamurugan, R.; Dekker, F. J.; Waldmann, H. Design of Compound Libraries Based on Natural Product Scaffolds and Protein Structure Similarity Clustering (PSSC) Mol. Biosyst. 2005, 1, 3645.

4 Newman, D. J.; Cragg, G. M.; Snader, K. M. Natural Products as Sources of New Drugs over the Period 1981-2002 J. Nat. Prod. 2003, 66, 1022-1037.

5 Clardy, J.; Walsh, C. Lessons from Natural Molecules Nature 2004, 432, 829-837.

6 Jaroszewski, J. W. Hyphenated NMR Methods in Natural Products Research, Part 1: Direct Hyphenation Planta Med. 2005, 71, 691-700. 
Jaroszewski, J. W. Hyphenated NMR Methods in Natural Products Research, Part 2: HPLC-SPENMR and Other New Trends in NMR Hyphenation Planta Med. 2005, 71, 795-802.

König, G. M.; Kehraus, S.; Seibert, S. F.; Abdel-Lateff, A.; Müller, D. Natural Products from Marine Organisms and Their Associated Microbes ChemBioChem 2006, 7, 229-238.

Andersen, R. J.; Faulkner, D. J.; Cun-heng, H.; Van Duyne, G. D.; Clardy, J. Metabolites of the Marine Prosobranch Mollusc Lamellaria sp. J. Am. Chem. Soc. 1985, 107, 5492-5495.

11 Cironi, P.; Albericio, F.; Álvarez, M. Lamellarins: Isolation, activity and synthesis Progress in Heterocyclic Chemistry. Volume 16. (G. W. Gribble and J. A. Joule, eds.) Pergamon: Oxford (UK), 2004, pp. 1-26.

Bailly, C. Lamellarins, From A to Z: A Family of Anticancer Marine Pyrrole Alkaloids Curr. Med. Chem.: Anti-Cancer Agents 2004, 4, 363-378.

13 Facompré, M.; Tardy, C.; Bal-Mahieu, C.; Colson, P.; Pérez, C.; Manzanares, I.; Cuevas, C.; Bailly, C. Lamellarin D: A Novel Inhibitor of Topoisomerase I Cancer Res. 2003, 63, 7392-7399.

14 Vanhuyse, M.; Kluza, J.; Tardy, C.; Otero, G.; Cuevas, C.; Bailly, C.; Lansiaux, A. Lamellarin D: A Novel Pro-Apoptotic Agent From Marine Origin Insensitive to P-Glycoprotein-Mediated Drug Efflux Cancer Lett. 2005, 221, 165-175.

15 Staker, B. L.; Hjerrild, K.; Feese, M. D.; Behnke, C. A.; Burgin, A. B., Jr.; Stewart, L. The Mechanism of Topoisomerase I Poisoning by a Camptothecin Analog Proc. Natl. Acad. Sci. U.S.A. 2002, 99, 15387-15392.

16 Marco, E.; Laine, W.; Tardy, C.; Lansiaux, A.; Iwao, M.; Ishibashi, F.; Bailly, C.; Gago, F. Molecular Determinants of Topoisomerase I Poisoning by Lamellarins: Comparison with Camptothecin and Structure-Activity Relationships J. Med. Chem. 2005, 48, 3796-3807. 
17 Ishibashi, F.; Tanabe, S.; Oda, T.; Iwao M. Synthesis and Structure-Activity Relationship Study of Lamellarin Derivatives J. Nat. Prod. 2002, 65, 500-504.

18 Tardy, C.; Facompré, M.; Laine, W.; Baldeyrou, B.; García-Gravalos, D.; Francesch, A.; Mateo, C.; Pastor, A.; Jiménez, J. A.; Manzanares, I.; Cuevas C.; Bailly, C. Topoisomerase I-Mediated DNA Cleavage as a Guide to the Development of Antitumor Agents Derived From the Marine Alkaloid Lamellarin D: Triester Derivatives Incorporating Amino Acid Residues Bioorg. Med. Chem. 2004, 12, 1697-1712.

19 Olsen, C. A.; Parera, N.; Albericio, F.; Álvarez, M. 5,6-Dihydropyrrolo[2,1-a]isoquinolines as Scaffolds for Synthesis of Lamellarin Analogues Tetrahedron Lett. 2005, 46, 2041-2044.

20 For clarity in this chapt the numbering of the lamellarins and scaffold is as in reference 15.

21 Heim, A.; Terpin, A.; Steglich, W. Biomimetic Synthesis of Lamellarin G Trimethyl Ether Angew. Chem. Int. Ed. 1997, 109, 155-156.

22 Ishibashi, F.; Miyazaki, Y.; Iwao, M. Total Synthesis of Lamellarin D and H. The First Synthesis of Lamellarin-Class Marine Alkaloids Tetrahedron 1997, 53, 5951-5962.

23 Banwell, M.; Flynn, B.; Hockless, D. Convergent Total Synthesis of Lamellarin K Chem. Commun. 1997, 259-260.

24 Cironi, P.; Manzanares, I.; Albericio, F.; Álvarez, M. Solid-Phase Total Synthesis of Pentacyclic System Lamellarins U and L Org. Lett. 2003, 5, 2959-2962.

25 Marfil, M.; Albericio, F.; Álvarez, M. Solid-Phase Synthesis of Lamellarins Q and O Tetrahedron 2004, 60, 8659-8668.

26 Cironi, P.; Cuevas, C.; Fernando Albericio, F.; Álvarez, M. Gaining Diversity in Solid-Phase Synthesis by Modulation of Cleavage Conditions From Hydroxymethyl-Based Supports. Application to Lamellarins Tetrahedron 2004, 60, 8669-8675. 

Lamellarin D J. Org. Chem. 2005, 70, 8231-8234. Lamellarins D, L, and H. Tetrahedron 2006, 62, 594-604.

29 An advantage of the protection was the increase in solubility of the compounds throughout the synthetic process, as well as, the prevention of undesired processes.

30 4d was not used as a building block. This entry is in the table for introducing the substituents of compounds 6d, 8d, and 10d.

31 (a) Kranenburg, M.; van der Burgt, Y. E. M.; Kamer, P. C. J.; van Leeuwn, P. W. N. M.; Goubitz, K. Fraange. New Diphosphine Ligands Based on Heterocyclic Aromatics Inducing Very High Regioselectivity in Rhodium-Catalyzed Hydroformylation: Effect of the Bite Angle $J$. Organometallics 1995, 14, 3081-3089. (b) Wolfe, J. P.; Singer, R. A.; Bryant, H. Y.; Buchwald, S. L. Highly Active Palladium Catalysts for Suzuki Coupling Reactions J. Am. Chem. Soc. 1999, $121,9550-9561$.

32 To obtain $\mathbf{2}, \mathbf{3}$ and $\mathbf{8}$, the protection of the phenolic groups is crucial to avoid byproducts during bromination.

33 Regioselectivity on the bromination of $\mathbf{6}$ to give $\mathbf{8}$ was checked easily by the absence of the singlet at $6.7 \mathrm{ppm}$, characteristic of $\mathrm{H}-2$.

34 Lower reaction time than for the less electron-rich analogs or lower reaction temperature did not improve the results.

35 In a previous study on the preparation of Lam-D (ref. 27), an excess of 6 equivalents of boronate were used; however, the reduction of that amount to 3 equivalents did not produce a significant change in the reaction yield. 
Alternatively, a more convergent synthesis of diarylated-compounds $\mathbf{9}$ with a range of substituted phenyl rings was attempted by regioselective Suzuki cross-coupling reaction on the dibromoscaffold 3. However, our first studies using an equimolar amount of the boronic building block $\mathbf{4 g}$ by the same reaction conditions as before produced $75 \%$ of a monoarylated bromide by HPLC-MS. Nevertheless, the 1H-NMR analyses evidenced the presence of an equimolecular amount 1-aryl- and 2-aryl-bromides and therefore the absence of regioselectivity.

Sotomayor, N.; Domínguez, E.; Lete. E. Oxidation Reactions of 2'-Functionalized 3Aryltetrahydro and 3,4-Dihydroisoquinolines Tetrahedron 1995, 51, 12721-12730.

Bermejo, A.; Andreu, I.; Suvire, F.; Leonce, S.; Caignard, D. H.; Renard, P.; Pierre, A.; Enriz, R. D.; Cortes, D.; Cabedo, N. Syntheses and Antitumor Targeting G1 Phase of the Cell Cycle of Benzoyldihydroisoquinolines and Related 1-Substituted Isoquinolines J. Med. Chem. 2002, 45, 5058-5068.

39 It was not possible to oxidize the scaffold $\mathbf{1}, \mathbf{6} \mathbf{1}$, and $\mathbf{7 1}$ using this procedure.

40 Both double doublets were assigned by gHSQC to C5"-H. See the gHSQC of $\mathbf{1 2 f}$ in the Supporting Information.

41 Semiempirical method PM3 was used for the energy minimization of each rotamer.

43 Concomitant demethylation of 4-methoxygroup occurred using an excess of 2.6 equivalents of $\mathrm{AlCl}_{3}$ when rich electron-ring building block as 3,4,5-trimethoxyphenyl was introduced to give for instance $14 \mathbf{a}\left(\mathrm{R}^{4}=\mathrm{OH}\right)$ and $\mathbf{1 8 c}\left(\mathrm{R}^{8}=\mathrm{OH}\right)$ with yield of $58 \%$ and $96 \%$, respectively. This demethylation was avoided using 1.3 equivalents of $\mathrm{AlCl}_{3}$ in $\mathbf{1 6 a}$ and $\mathbf{1 7 a}$.

44 The letter numbershooter for the compounds 13-18 are the same as indicated in Table 1 and takes account the deprotection of $i \mathrm{PrO}$-groups $\left(\mathrm{R}^{3}, \mathrm{R}^{4}, \mathrm{R}^{6}\right.$, and $\left.\mathrm{R}^{8}\right)$ to give $\mathrm{OH}$. 
(a) Skehan, P.; Storeng, R.; Scudiero, D.; Monks, A.; McMahon, J.; Vistica, D.; Waren, J. T.;

Bokesch, H.; Kenney, S.; Boyd, M. R. New colorimetric cytotoxicity assay for anticancer Drug Screening. J. Natl. Cancer Inst., 1990, 82, 1107-1112. (b) Faircloth, G. T.; Stewart, D.; Clement, J. J. A simple screening procedure for the quantitative measurement of cytotoxicity assay. $J$. Tissue Cult. Methods 1988, 11, 201-205. 
Table 1

\begin{tabular}{|c|c|c|c|c|c|c|c|c|c|c|c|}
\hline 4 & $\mathbf{R}$ & $\mathbf{R}^{2}$ & $\mathbf{R}^{3}$ & $\mathbf{R}^{4}$ & $\mathbf{R}^{5}$ & 5 & $\mathbf{R}^{6}$ & $\mathbf{R}^{7}$ & $\mathbf{R}^{8}$ & $\mathbf{R}^{9}$ & $\%$ \\
\hline $\mathbf{a}$ & $\mathrm{H}$ & $\mathrm{H}$ & $\mathrm{OMe}$ & $\mathrm{OMe}$ & $\mathrm{OMe}$ & $\mathbf{a}$ & $\mathrm{O} i \mathrm{Pr}$ & $\mathrm{H}$ & $\mathrm{O} i \mathrm{Pr}$ & $\mathrm{OMe}$ & 80 \\
\hline b & $\mathrm{H}$ & $\mathrm{H}$ & $\mathrm{H}$ & $\mathrm{OH}$ & $\mathrm{H}$ & b & $\mathrm{O} i \mathrm{Pr}$ & $\mathrm{H}$ & $\mathrm{O} i \mathrm{Pr}$ & $\mathrm{O} i \mathrm{Pr}$ & 52 \\
\hline c & $\mathrm{CMe}_{2} \mathrm{CMe}_{2}$ & $\mathrm{H}$ & $\mathrm{OMe}$ & $\mathrm{OH}$ & $\mathrm{H}$ & c & $\mathrm{O} i \mathrm{Pr}$ & $\mathrm{H}$ & $\mathrm{O} i \mathrm{Pr}$ & $\mathrm{H}$ & 64 \\
\hline d & ---- & $\mathrm{H}$ & $\mathrm{OMe}$ & $\mathrm{O} i \mathrm{Pr}$ & $\mathrm{H}$ & d & $\mathrm{O} i \mathrm{Pr}$ & $\mathrm{OMe}$ & $\mathrm{OMe}$ & $\mathrm{H}$ & 61 \\
\hline e & $\mathrm{H}$ & $\mathrm{OMe}$ & $\mathrm{H}$ & $\mathrm{H}$ & $\mathrm{OMe}$ & e & $\mathrm{OMe}$ & $\mathrm{H}$ & $\mathrm{O} i \mathrm{Pr}$ & $\mathrm{OMe}$ & 81 \\
\hline f & $\mathrm{H}$ & $\mathrm{H}$ & $\mathrm{OMe}$ & $\mathrm{OMe}$ & $\mathrm{H}$ & 9 & Scaf & old 8 & Bor & olane & $\%$ \\
\hline g & $\mathrm{H}$ & $\mathrm{H}$ & $\mathrm{H}$ & $\mathrm{OMe}$ & $\mathrm{H}$ & $\mathbf{a}$ & & & & $4 b$ & 76 \\
\hline $\mathbf{h}$ & $\mathrm{H}$ & $\mathrm{H}$ & $\mathrm{OMe}$ & $\mathrm{H}$ & $\mathrm{OMe}$ & b & & & & $4 \mathrm{ff}$ & 89 \\
\hline $\mathbf{i}$ & $\mathrm{H}$ & $\mathrm{H}$ & $\mathrm{H}$ & $\mathrm{OCF}_{3}$ & $\mathrm{H}$ & c & & & & $4 a$ & 71 \\
\hline $\mathbf{j}$ & $\mathrm{H}$ & $\mathrm{H}$ & $\mathrm{H}$ & $\mathrm{O} i \mathrm{Pr}$ & $\mathrm{H}$ & d & & & & $5 a$ & 89 \\
\hline $\mathbf{k}$ & $\mathrm{H}$ & $\mathrm{H}$ & $\mathrm{O} i \mathrm{Pr}$ & $\mathrm{H}$ & $\mathrm{H}$ & $\mathbf{e}$ & & & & $5 e$ & Quant. \\
\hline 1 & $\mathrm{H}$ & $\mathrm{H}$ & $\mathrm{H}$ & $\mathrm{NMe}_{2}$ & $\mathrm{H}$ & $\mathbf{f}$ & & & & $5 c$ & 82 \\
\hline m & $\mathrm{H}$ & $\mathrm{H}$ & $\mathrm{NO}_{2}$ & $\mathrm{H}$ & $\mathrm{H}$ & g & & & & $5 b$ & 81 \\
\hline $\mathbf{n}$ & $\mathrm{H}$ & \multicolumn{4}{|c|}{ 2-thienyl } & h & \multicolumn{2}{|c|}{$8 \mathbf{k}$} & \multicolumn{2}{|c|}{$5 c$} & 95 \\
\hline & & & & & & $\mathbf{i}$ & \multicolumn{2}{|c|}{$8 k$} & \multicolumn{2}{|c|}{$5 d$} & 93 \\
\hline
\end{tabular}


Table 2.

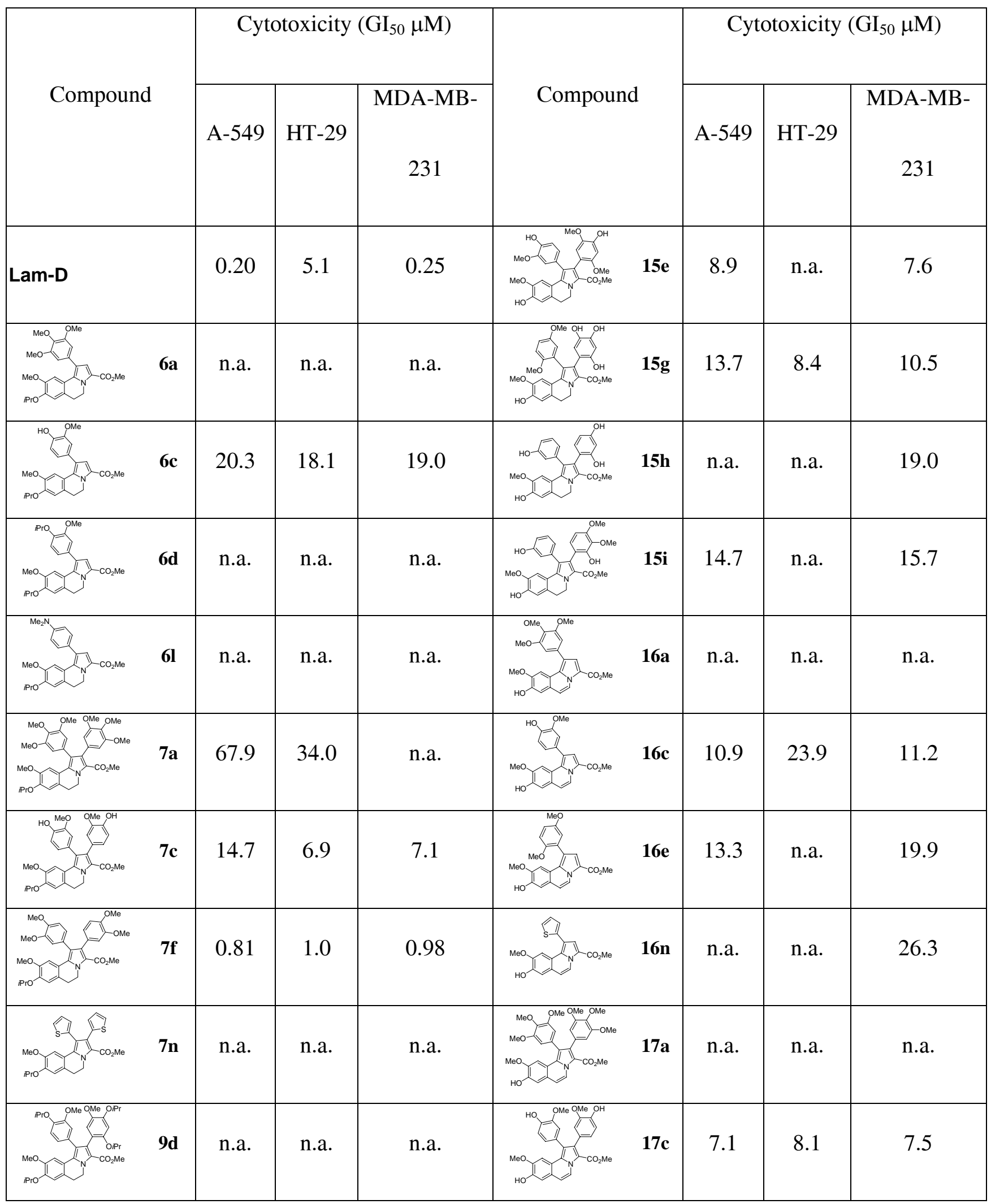




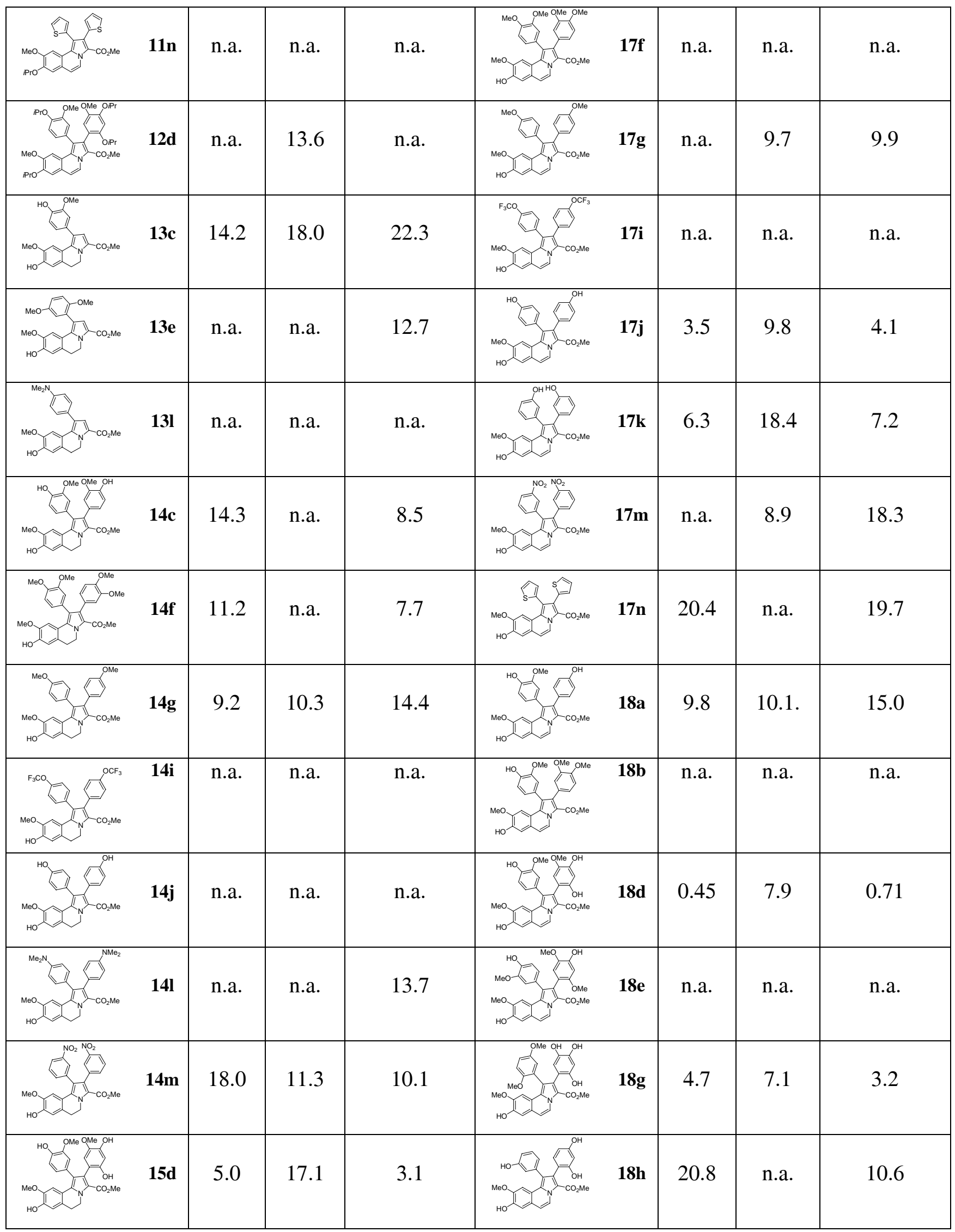

n.a. $=$ not active at $10 \mu \mathrm{g} / \mathrm{mL}$ 


\section{Figure 1.}
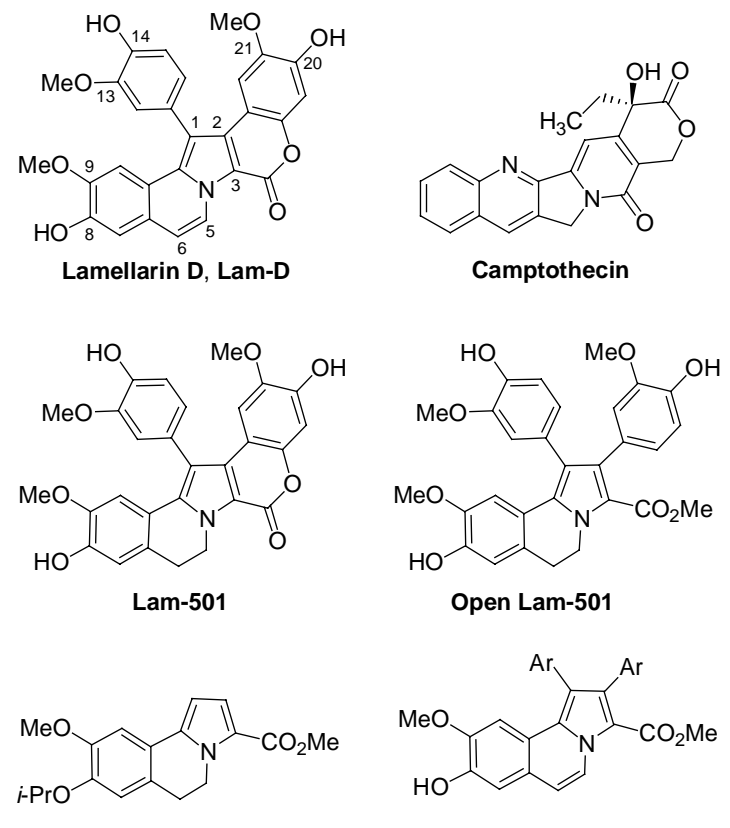

Scaffold, 1

Oxidized diaryl-scaffold

Figure 2.

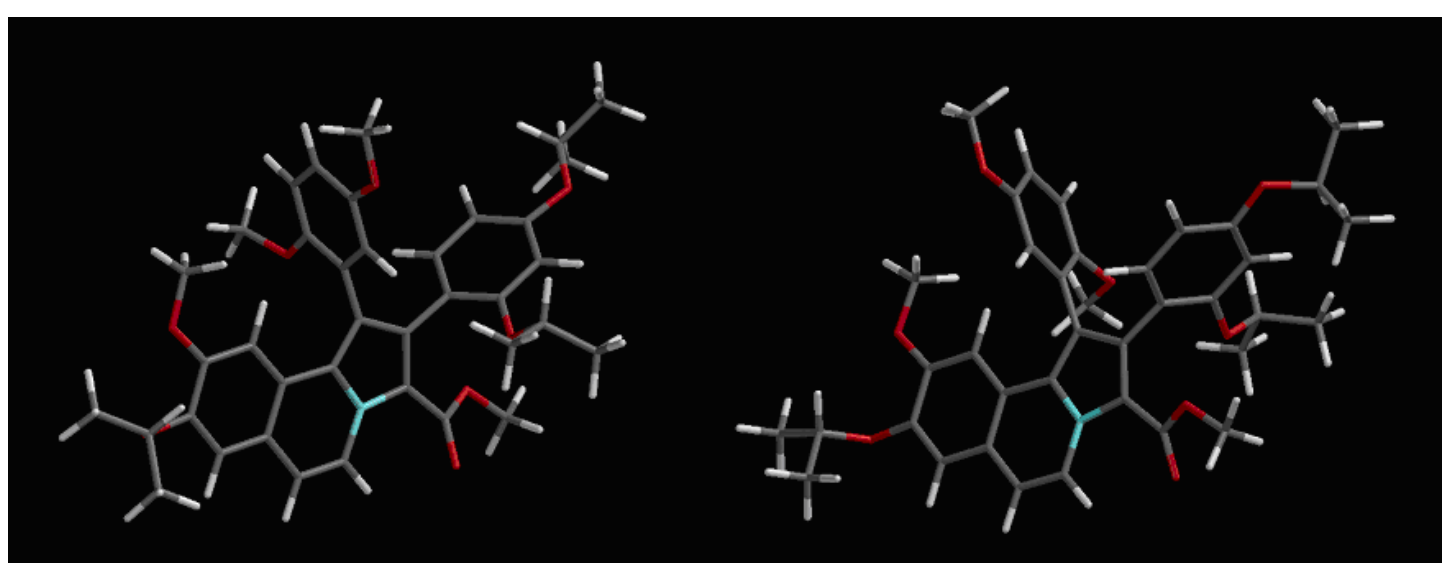


Scheme 1.

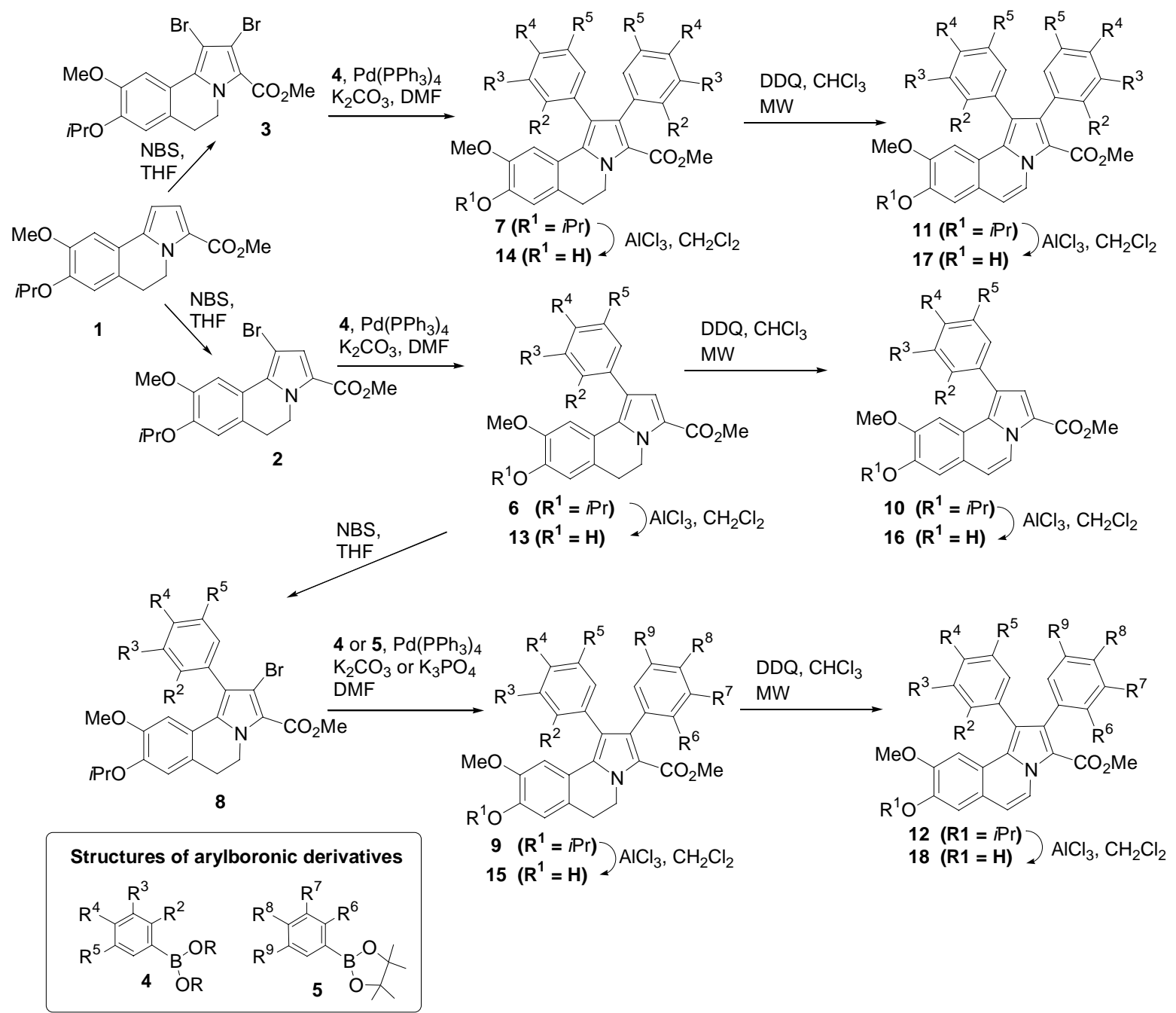




\section{Scheme Legends}

Table 1. Substituents of building blocks 4 and $\mathbf{5}$, and compounds 9

Table 2. In vitro cytotoxicity of the open-chain analogs of Lam-D and synthetic intermediates

Figure 1. Structures of lamellarins, camptotecin and scaffold 1

Figure 2. Minimized energy forms of the two rotamers of compound $\mathbf{1 2 f}$

Scheme 1. Synthesis of open-chain lamellarin analogs library

\section{TOC Graphic}
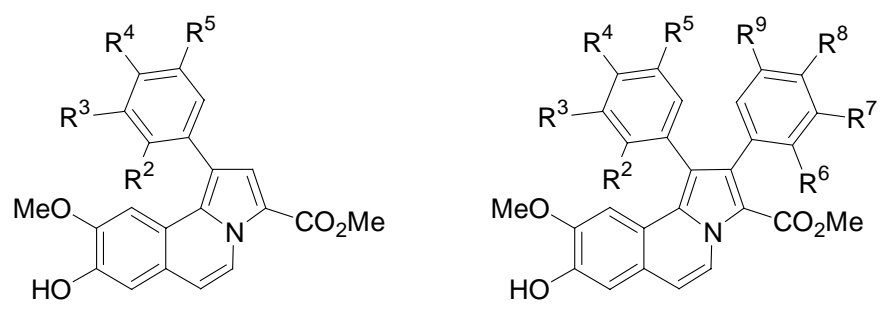